\title{
A uniform X-ray analysis of 79 distant galaxy clusters with ROSAT and ASCA ${ }^{\star}$
}

\author{
N. Ota ${ }^{1,2, \star \star}$ and K. Mitsuda ${ }^{2}$ \\ ${ }^{1}$ Department of Physics, Tokyo Metropolitan University, 1-1 Minami-osawa, Hachioji, Tokyo 192-0397, Japan \\ e-mail: ota@crab.riken.jp \\ 2 Institute of Space and Astronautical Science, Japan Aerospace Exploration Agency, 3-1-1 Yoshinodai, Sagamihara, \\ Kanagawa 229-8510, Japan \\ e-mail: mitsuda@astro.isas.jaxa.jp
}

Received 14 April 2004 / Accepted 22 July 2004

\begin{abstract}
We present a uniform analysis of the ROSAT HRI and the ASCA GIS/SIS data for 79 distant clusters of galaxies in the redshift range $0.1<z<0.82$ to study the global structures of the intracluster medium. We have constructed an X-ray catalog consisting of the largest sample of clusters in the redshift range for which pointed X-ray observations were carried out with both the observatories. We determined the emission-weighted X-ray temperatures of the clusters with ASCA, while we studied surface brightness distribution with the ROSAT HRI utilizing the isothermal $\beta$ model. We investigated the statistical properties and trends for redshift evolution of the X-ray parameters including the temperature, the density profile of the intracluster gas and the gas-mass fraction within $r_{500}$. We also present correlations of the cluster parameters with the X-ray temperature and with the core radius and compare them with the predictions of the self-similar model, from which we discuss the possible origin of the double structure discovered in the core radius distribution.
\end{abstract}

Key words. galaxies: clusters: general - galaxies: intergalactic medium - X-rays: galaxies - cosmology: observations cosmology: dark matter - catalogs

\section{Introduction}

Clusters of galaxies are the largest collapsed systems known in the universe. Because the time scale of evolution of clusters is a significant fraction of the age of the Universe, the clusters may preserve information about the early universe and thus are considered to be excellent tracers of the formation and evolution of structures. They are considered to continue to grow into larger systems through complex interaction between smaller systems, namely merging process. It is possible that the clusters that we see are in different stages of evolution.

For the understanding of cluster structure and evolution, we believe it is important to analyze systematically a large number of clusters at various redshifts. After the ASCA (Tanaka et al. 1994) and the ROSAT (Trümper 1993) X-ray observatories were put into orbit, it became possible to study relatively distant clusters at X-ray energies. During 7-10 years of observations, more than one hundred clusters were recorded with both observatories. Since ASCA has a high sensitivity to measure the $\mathrm{X}$-ray spectrum in the wide energy band while ROSAT is good at imaging in the soft X-ray band, the two observatories are an excellent combination to study properties of the

* All appendices and Tables 1-7 are only available in electronic form at http: //www. edpsciences.org

$\star \star$ Present address: Cosmic Radiation Laboratory, RIKEN, 2-1 Hirosawa, Wako, Saitama 351-0198, Japan. intracluster medium (ICM). At present the XMM-Newton and the Chandra satellites are in orbit and generate much cluster data with higher sensitivities. However, as we will mention below, the data set used in the present paper will be one of the best existing to construct the largest sample of distant clusters and study global X-ray structures.

$\mathrm{X}$-ray observations bring us valuable information on not only the physical state of the ICM but also the underlying potential structure of the clusters. Statistical studies are very powerful in exposing the physical nature of the clusters. In particular, nearby clusters have been extensively studied at $\mathrm{X}$-ray wavelengths. As to the low redshift samples $(z \lesssim 0.1)$, Mohr et al. (1999) performed a systematic analysis on the ROSAT PSPC data of 45 clusters and utilizing the published ICM temperatures, they investigated the correlation between the ICM mass and the temperature, namely the $M_{\text {gas }}-T$ relation. They found that the slope of the relation is significantly steeper than that predicted from the self-similar model (Kaiser 1986). The observed luminosity-temperature $\left(L_{X}-T\right)$ relation is also known to be steeper than the expectation of the selfsimilar model (e.g. David et al. 1993). The inconsistency between the observations and the simple theoretical model has been debated for many years and various possibilities such as non-gravitational heating (e.g. Evrard \& Henry 1991; Cavaliere et al. 1997) and dependence of gas mass or gas-mass fraction on the temperature have been proposed (e.g. David et al. 1993; 
Neumann \& Arnaud 2001). For higher redshift samples, however, the number of clusters that were uniformly analyzed was limited compared to the nearby clusters. The ASCA spectroscopic data of distant clusters were compiled by Mushotzky $\&$ Scharf (1997) (38 clusters with $z>0.14$ ), White (2000) (41 clusters with $z>0.1$ and 65 clusters with $z<0.1$ ), and Novicki et al. (2002) (32 clusters with $0.3<z<0.6$ and 53 clusters with $z<0.3$ ), while the combined (i.e. spectral and imaging) analyses of the distant clusters were separately published in Allen (1998) (13 clusters with $0.1<z<0.45$ ), Hashimotodani (1999) (27 clusters with $0.1<z<0.78$ ), Ettori \& Fabian (1999) (36 clusters at $z>0.05$ ), Schindler (1999) (11 clusters at $z>0.3$ ), Lewis et al. (1999) (14 clusters with $0.14<z<0.55$ ) and Vikhlinin et al. (2002) (22 clusters at $z>0.4$ ). Recently, Ettori et al. (2004) reported the Chandra analysis of 28 clusters at $0.4<z<1.3$ and observed the steeper slopes in the $L-T$ and the $M_{\text {gas }}-T$ relations, which provided hints of negative evolution in their relations at high redshift.

On the other hand, Ota (2001) and Ota \& Mitsuda (2002) have attempted to construct the largest X-ray sample of distant clusters with $z>0.1$ based on the combined analysis of the ROSAT HRI and the ASCA GIS/SIS data. The data set used in the analysis is one of the best suited for the purpose of the present systematic study for the following reasons: the largest cluster sample of the pointed observations were stored in the ROSAT and the ASCA archival database, which enable us to cover the widest redshift range up to $z \sim 1$. The focal plane instruments, the ROSAT HRI and the ASCA GIS/SIS have sufficient sensitivities to study the global properties of the ICM spatial structure with a typical resolution of $5^{\prime \prime}$ and spectral features such as the ICM temperature and the luminosity, respectively. In addition, the instruments' responses were well calibrated and the use of the same detectors for all the clusters will reduce systematic effects when comparing their results. Our sample covers the intermediate redshift range of $0.1-0.8$ between those of Mohr et al.'s sample $(z<0.1)$ and Ettori et al.'s sample $(0.4<z<1.3)$, thus combining our data with the other two samples is helpful in discussing the evolution of internal structure of the ICM directly from the observational point of views.

In this paper we describe a uniform analysis of the ROSAT HRI and the ASCA SIS/GIS data of 79 clusters performed in Ota (2001) and Ota \& Mitsuda (2002) and thus provide an $\mathrm{X}$-ray database of ICM structure with the widest redshift range of $0.1<z<0.82$. In order to perform a reliable parameter determination we paid special attention to evaluating all possible systematic errors in the spatial and the spectral analyses. We determined the spectral and spatial properties of the intracluster gas in terms of the temperature, the core radius and the central electron density etc. for all of the clusters. In the image analysis we found that the sample can be naturally classified into regular and irregular clusters according to the X-ray surface brightness distribution. We present the statistical properties of the X-ray parameters and the relations to the double- $\beta$ nature of the clusters discovered in the core radius distribution. We also estimated the cluster total mass, the gas mass and the gasmass fraction within a radius corresponding to a fixed overdensity of 500. We further studied the redshift dependency of the parameters and their correlations, where we considered a systematic error due mainly to the limited sensitivity of the instruments and some assumptions used in the estimations.

This paper is organized in the following manner. In the next section, we describe the characteristics of our samples. In Sects. 3 and 4, we explain the methods of spatial analysis with ROSAT and spectral analysis with ASCA in detail. In Sect. 5, we derive the X-ray parameters to describe the global structure of clusters and investigate their redshift dependence. In Sect. 6 we study correlations of the parameters and discuss the properties of the ICM. In Sect. 7 we summarize our results.

We use $\Omega_{\mathrm{M}}=0.3, \Omega_{\Lambda}=0.7$ and $h_{70} \equiv$ $H_{0} /\left(70 \mathrm{~km} \mathrm{~s}^{-1} \mathrm{Mpc}^{-1}\right)=1$. The quoted errors are the $90 \%$ confidence range throughout the paper except where noted.

\section{The sample}

We have selected distant clusters with $0.1 \leq z \leq 1$ for which pointed X-ray observation data with both ASCA and ROSAT/HRI are available. Though there are 83 clusters which meet the criteria, we rejected three (A222, A223, A1758S) because of the large $\left(>210^{\prime \prime}\right)$ pointing off-axis angles in the ROSAT observations. We did not include a high-redshift cluster, AXJ2016+112 at $z=1$ (Hattori et al. 1997) because the Chandra observation showed that the X-ray emission from the direction of AXJ2016+112 can be resolved into point sources and the cluster diffuse emission is not prominent (Chartas et al. 2001). The final sample comprises 79 clusters. Among them, three clusters (\#1 PKS 0745-19, \#6 A2204, and \#13 A1689) overlap with Mohr et al. (1999)'s nearby cluster sample and thirty are known to have strong gravitational lensing signals (e.g. Hattori et al. 1999). The observation logs are summarized in Table 1, where the clusters were sorted according to the redshift and numbered from 1 to 79 . The redshift distribution of the sample is shown in Fig. 1a. The clusters with $0.1 \leq z<0.3$, $0.3 \leq z<0.5$ and $0.5 \leq z<1$ make up 58\%, 32\%, and $10 \%$ of the sample, respectively. The average redshift is 0.30 .

Because our analysis targets were collected from the proposal observations and the sensitivities for high-redshift clusters are limited, we have to carefully consider possible selection bias. In the first step, we compare the sample with other unbiased cluster samples. We show temperature distributions of our sample and the nearby X-ray flux-limited 55 cluster sample constructed by Edge et al. (1990) in Fig. 2. Our sample covers the equivalent temperature range, but has a higher average temperature of $6.8 \mathrm{keV}$. The Kolmogorov-Smirnov (K-S) test gave the probability that the two samples are from the same temperature distribution as 0.06 (the $\mathrm{K}-\mathrm{S}$ parameter, $D=0.24$ ). Observation bias will be discussed in Sects. 5.1.1 and 5.2.2 in more detail.

\section{Spatial analysis}

\subsection{Data reduction from event lists}

We have retrieved the event lists of the ROSAT HRI detector from the ROSAT Data Archive of the Max-Plank-Institut für extraterrestrische Physik (MPE) at Garching, Germany. We used 

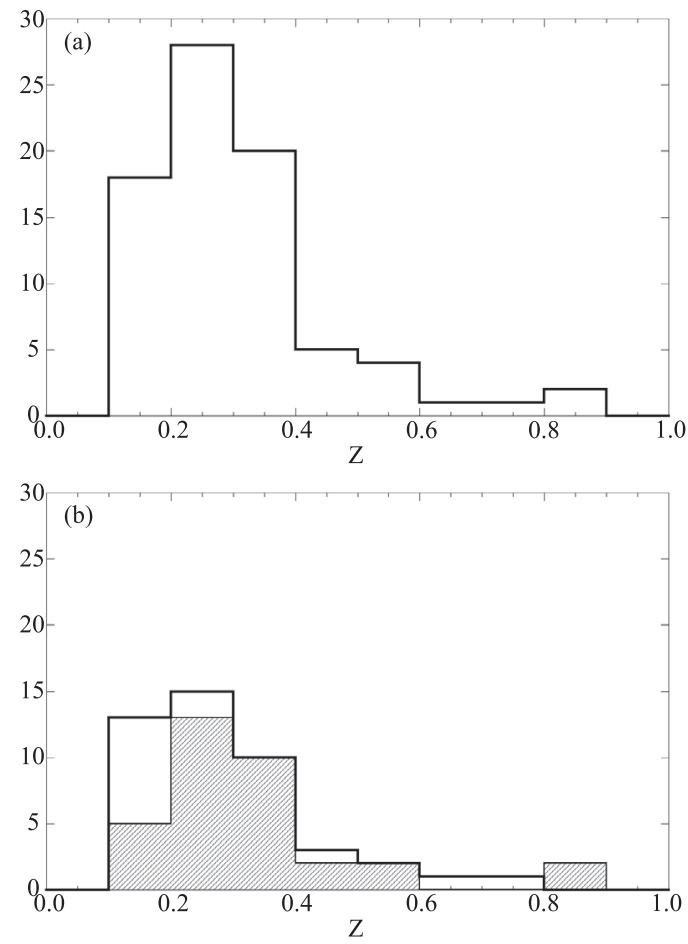

Fig. 1. a) Redshift distribution of 79 distant clusters. b) Redshift distribution of the 45 regular clusters (open) and the 34 irregular clusters (hatched).

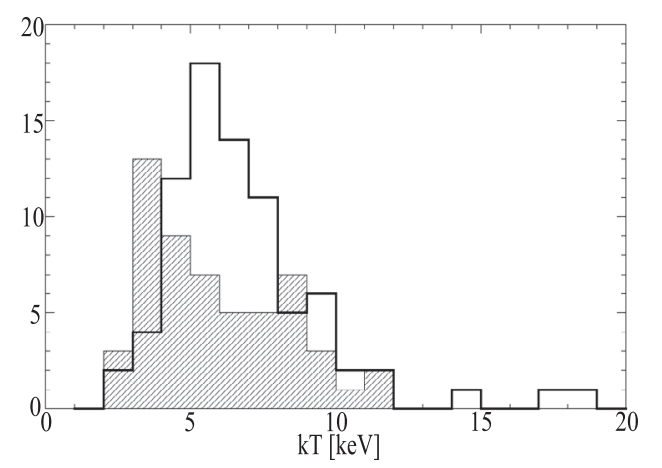

Fig. 2. Comparison of the temperature distribution of our 79 distant clusters (open) to the nearby flux limited 55 sample of Edge et al. (1990) (hatched).

the EXSAS analysis package (Zimmermann et al. 1992) to produce X-ray images from the event lists. The raw HRI data has a 0 .'5 spatial resolution. However, since the half power diameter of the X-ray telescope is $4 . \prime 8$ at the optical axis and the photon counting statistics of the present data are limited, it is not worth oversampling the telescope point spread function (PSF). We thus rebinned the image into $5^{\prime \prime}$ bins, within whose diameter about $70 \%$ of photons from a point source are included.

If there were multiple pointings for a cluster, and the pointing offset angles between them are smaller than $3^{\prime}$, we superpose the event lists in the sky coordinate. The PHA channel of $1-10$, which corresponds to $0.2-2 \mathrm{keV}$, was used to avoid particle background events.

Next we searched for foreground/background sources in the field of view with the standard source detection program in the
EXSAS. We created lists of all the sources detected by the maximum likelihood technique and having a likelihood of $>8$. We used the lists to exclude those sources from the analysis.

\subsection{Centroid determination and $X$-ray morphology}

We apply a method to determine cluster centroids and classify the morphology of clusters. A similar technique to find the cluster center was first introduced by Mohr et al. (1993). We extended the method to evaluate the systematic errors of the centroid determination for distant clusters and found that the analysis can also be used to determine the regularity of the $\mathrm{X}$-ray distribution. The cluster emission is typically extended about $4^{\prime}$ from the optical axis of the telescope. In this image region, the position dependence of the telescope vignetting is known to be less than $2 \%$ (Briel et al. 1997). The position dependence of the background intensity is also small up to $\sim 12^{\prime}$. Thus they do not affect the centroid determination.

The analysis consists of two major steps. First we estimate the 0th order center position and a parameter which represents the extent of the X-ray image, utilizing 1-dimensional image projections and Gaussian fits to them. Then, starting from the Oth order initial value, we determine the center from the center of gravity of the photon distribution.

First, we extracted a $4^{\prime} \times 4^{\prime}$ image that contains the central region of the cluster emission. Then we projected along the $x$ - and $y$-axes to get one-dimensional intensity profiles. We fit these with Gaussian functions, and determined the mean, $\left(x_{G, 0}, y_{G, 0}\right)$ and the width, $\left(\sigma_{x, 0}, \sigma_{y, 0}\right)$. To determine these values with higher accuracy, we extracted an image of size $3 \sigma_{x, 0} \times 3 \sigma_{y, 0}$ whose center is at $\left(x_{G, 0}, y_{G, 0}\right)$ and performed the one-dimensional Gaussian fitting again to derive the next set of $\left(x_{G, 1}, y_{G, 1}\right)$ and $\left(\sigma_{x, 1}, \sigma_{y, 1}\right)$. The procedure was iterated $i$ times until the mean position converged within 0.1 pixels i.e. $\left|\left(x_{G, i}, y_{G, i}\right)-\left(x_{G, i-1}, y_{G, i-1}\right)\right|<(0.1,0.1)$ or the number of iterations reached $i=20$. We then define a parameter $\bar{\sigma}$ as $\bar{\sigma} \equiv\left(\sigma_{x, i}+\sigma_{y, i}\right) / 2$, which is a measure of the image extent for further analysis.

Next we determine the cluster center from the center of gravity of the photon distribution in an aperture circle of radius, $R$. Then if the $\mathrm{X}$-ray image is circularly symmetric and the center of the circle is at the X-ray center, the center of gravity of the photon distribution should coincide with the center of the circle. For a given value of $R$, we can determine the center position, $\boldsymbol{r}_{i} \equiv \sum_{R} \boldsymbol{p} / \sum_{R} 1$, where $\boldsymbol{p}$ represents the position of the photon, and $\sum_{R}$ sums all the photons over the circular area of the radius $R$. Then starting with the mean position determined in the previous paragraph, $\boldsymbol{r}_{0}=\left(x_{G, i}, y_{G, i}\right)$, we extract a circular image of the radius $R$ centered at $\boldsymbol{r}_{0}$, and calculate the centroid position $\boldsymbol{r}_{1}$. We continue the iteration until $\left|\boldsymbol{r}_{i}-\boldsymbol{r}_{i-1}\right|$ becomes less than 0.1 pixels. If there were contaminating sources in the circle, we excluded the region centered at the sources and the region symmetric to them so as not to affect the centroid determination. We changed the aperture radius $R$ from $2 \bar{\sigma}$ to $9 \bar{\sigma}$ to study the $R$ dependence of the results. As a result, while some clusters showed constant centroids almost independent of the 

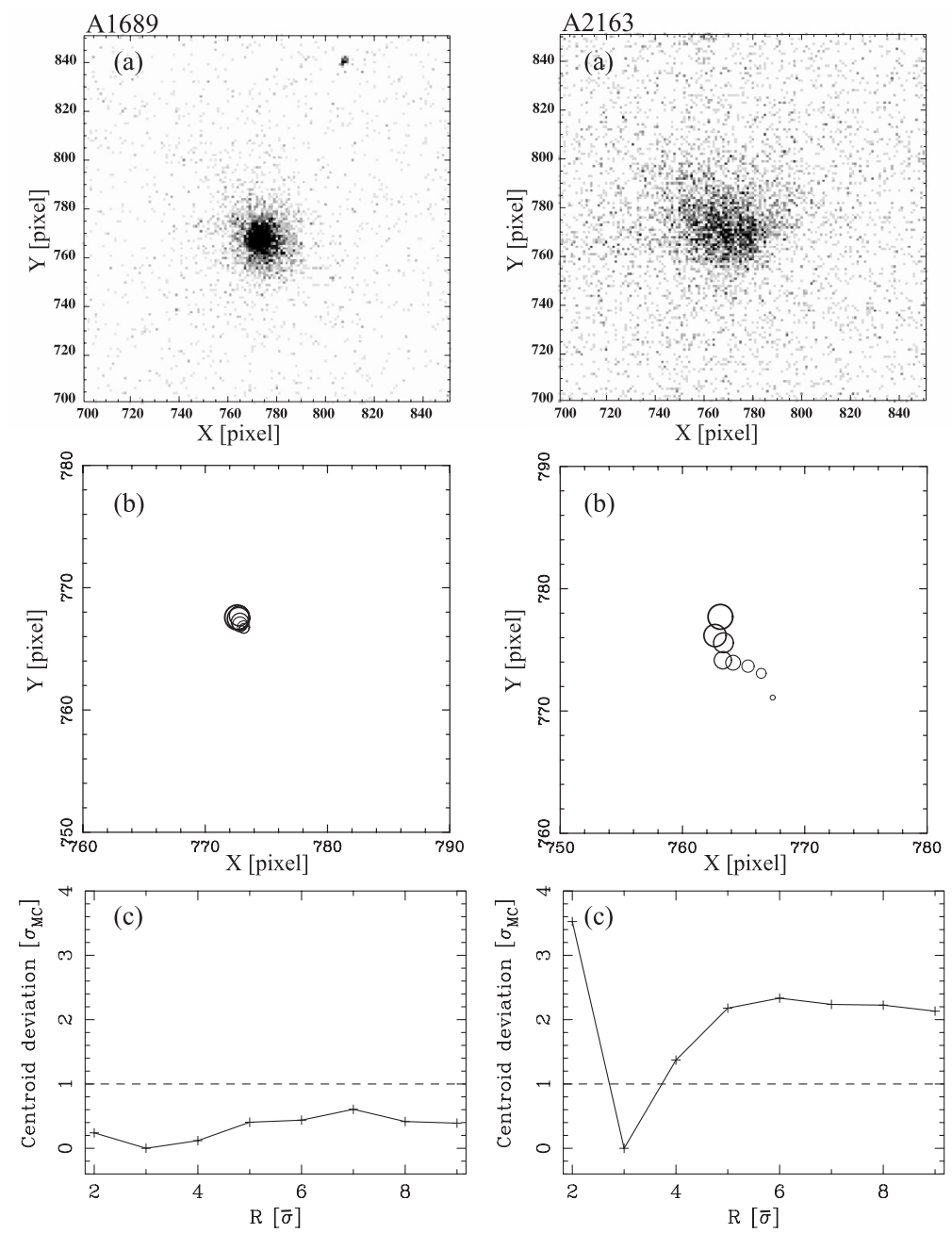

Fig. 3. Centroid determination and classification of X-ray morphology. \#13 A1689 and \#19 A2163 are shown in the left and right panels, respectively. From top to bottom: a) the HRI image in the pixel coordinates. b) The centroids determined in circles of radii $R=n \bar{\sigma}(n=$ $2,3, . ., 9$ ), where the size of the symbols are nearly proportional to $n$. c) The deviations the centroids relative to that determined for $3 \bar{\sigma}$ in units of standard error, shown as a function of $R[\bar{\sigma}]$. While A1689 is classified as a regular cluster, A2163 is an irregular cluster due to the significant centroid deviation.

radius, others exhibited systematic behavior. Two representative cases are shown in Fig. 3.

The dependence of the center of gravity on the aperture radius may indicate some asymmetry or substructures of the cluster. However, because the results with different $R$ are not statistically independent, the Poisson fluctuations may produce systematic deviations. To estimate this effect, we performed Monte-Carlo simulations and generated a number of simulation images assuming the isothermal $\beta$-model as the X-ray image distribution. We calculated the RMS value of the distance between the center of gravity and the true center, $\sigma_{\mathrm{MC}}$, for various combinations of parameters, the cluster core radius $r_{\mathrm{c}}$, the number of photons $N$ and the size of the aperture $R$, while $\beta$ was fixed to the typical value of 0.67 .

If the displacement of the center of gravity with different values of the aperture radius $R$ is larger than the standard deviation determined from the simulation, we can conclude that there is asymmetry or substructures. In Fig. 3, we show the deviation of the center of gravity from that determined for $R=3 \bar{\sigma}$ normalized by the standard deviation. For some clusters the deviation is well within the $1 \sigma_{\mathrm{MC}}$ level; however, for others it is not. We define criteria for irregularity of clusters as follows: (1) more than 1 data point whose deviation exceeds $3 \sigma_{\mathrm{MC}}$, and/or (2) deviations ( $>1 \sigma_{\mathrm{MC}}$ ) seen in more than a few consecutive points. Accordingly, we classified our sample into regular and irregular clusters. The results are listed in Table 2 . The ratio of regular to irregular is 45:34 and the redshift distributions of the two subgroups are shown in Fig. 1b.

\subsection{Radial X-ray surface brightness profiles}

We derive azimuthally-averaged radial profiles of the X-ray surface brightness centered at the cluster centroids that were determined within the $3 \bar{\sigma}$ aperture radii for both the regular and irregular clusters. We have chosen the centroids because they are less affected by the Poisson fluctuations in the outer image regions. The bin size of the radial profile is $5^{\prime \prime}$. We excluded the contaminating sources from the integration area with a circle of radius 5 times the FWHM of the PSF at position. 
We study the effect of the choice of the center on the results of the radial profile fits, by shifting the center positions determined for $R$ between $2 \bar{\sigma}$ and $9 \bar{\sigma}$. We found the $\beta$-model parameters (Sect. 3.4) constant within the statistical errors for all the regular clusters and many of the irregular clusters. For $30 \%$ of the irregular clusters, the resulting core radius increases typically by a factor of 2 as $R$ increases from $2 \bar{\sigma}$ to $9 \bar{\sigma}$.

\subsection{Radial profile fitting with a single $\beta$-model}

In the following two subsections, we analyze the X-ray radial profile under the isothermal $\beta$-model (Cavaliere \& Fusco-Femiano 1976). The single $\beta$-model fitting function is written as

$S(r)=S_{0}\left[1+\left(\frac{r}{r_{\mathrm{c}}}\right)^{2}\right]^{-3 \beta+1 / 2}+C$,

where $S_{0}, r_{\mathrm{c}}$ and $\beta$ are the central surface brightness, core radius and the outer slope, respectively, and $C$ is a constant background. In Fig. 4a we show an example of the radial profile fit.

It is crucial for reliable determination of the model parameters to estimate the background level correctly. The HRI background is dominated by the particle background (Briel et al. 1997) and the detailed calibration by Snowden (1998) showed that it can be modeled by a constant image within $\sim 12^{\prime}$ from the detector center. The counting rate of the particle background depends strongly on the satellite orbit and time, and typically ranges from 1 to 10 counts s$^{-1}$ over the entire detector. Thus the background counting rate needs to be determined observation by observation. We determined the background level from the radial profile including the background as one of the fitting parameters as Eq. (1). Since we do not know the true extent of the cluster emission and also $r_{\mathrm{c}}$ and $\beta$ are strongly coupled (Fig. 5), the background level and the model parameters depend on the outer radius of the fitting area, $r_{\text {out }}$. Particularly when $r_{\text {out }}$ is too small, the background level determined from the fit tends to be over- or under-estimated and results in uncertain $r_{\mathrm{c}}$ and $\beta$. However, as shown in Fig. 6, the best-fit parameters converge to constants if $r_{\text {out }}$ is large enough, typically $\gtrsim 10 \bar{\sigma}$. For all the clusters, we confirmed that the parameters converge at $r_{\text {out }}=12^{\prime}$. Thus we decided to adopt this value for all clusters, within which the background can be regarded as constant. Finally the background level we obtained from fits are reasonable background levels.

Because the effective area of the X-ray telescope decreases with off-axis angle, the cluster image may be affected by the vignetting. However since the off-axis angle of the cluster centroid of the present data is smaller than $4^{\prime}$, and the typical spatial extent of the present clusters is $\sim 4^{\prime}$, the vignetting effect is at most $3 \%$ at the rim of the clusters (the HRI vignetting function is given in Briel et al. (1997)). Although we performed fits with the $\beta$-model fitting function with and without the correction of the vignetting function, the results showed no difference.

The $\beta$-model function needs to be convolved with the X-ray telescope PSF, then integrated over the image bin. However,

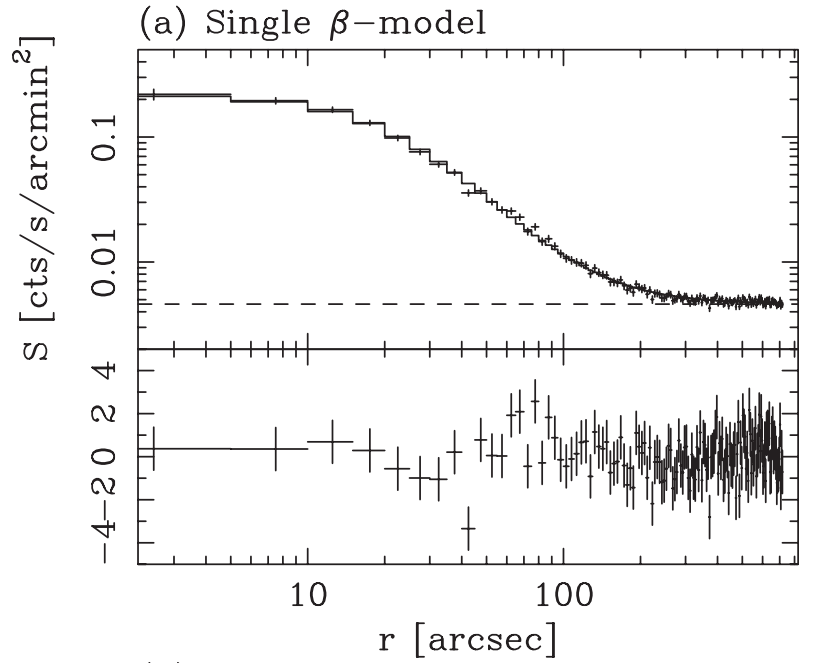

(b) Double $\beta$-model

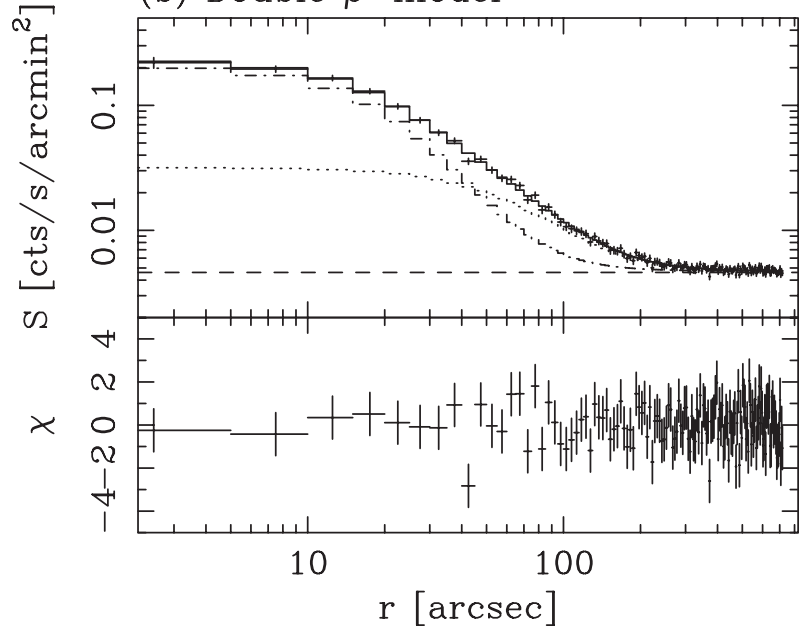

Fig. 4. Radial X-ray surface brightness profile of \#13 A1689 fitted with the single $\beta$-model a) and the double $\beta$-model b). The crosses denote the observed radial profile of the ROSAT HRI, and the step functions show the best-fit $\beta$-models. The best-fit background levels are shown with the horizontal dashed lines. In the panel b), the contribution of the inner and the outer component of the double $\beta$-model are also shown with the dash-dot and the dotted lines, respectively.

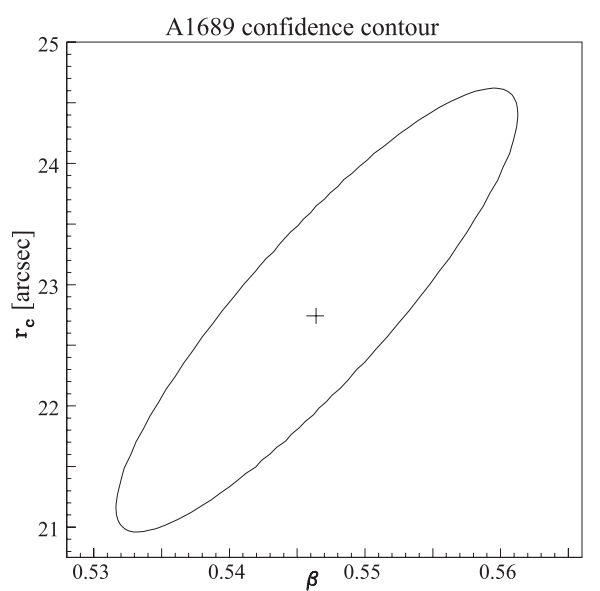

Fig. 5. $\chi^{2}$ contour of the single $\beta$-model fit for \#13 A1689. The position of $\chi^{2}$ minimum is denoted with a cross. The curve corresponds to the single-parameter error domain at $90 \%$ confidence. 


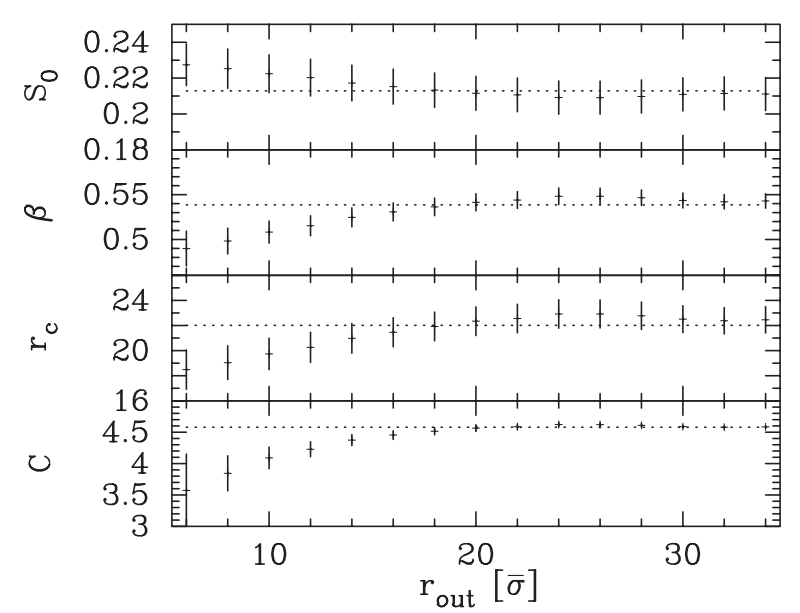

Fig. 6. Effect of the outer cut off radius in the single $\beta$ model fitting. The results for \#13 A1689 are shown as an example. The four parameters of the single $\beta$-model, $S_{0}$ [counts s $\operatorname{arcmin}^{-2}$ ], $\beta, r_{\mathrm{c}}$ [arcsec], $C\left[10^{-3}\right.$ counts s$\left.^{-1} \operatorname{arcmin}^{-2}\right]$ are shown as a function of the outer cutoff radius, $r_{\text {out }}$ in unit of $\bar{\sigma}$. The results of the constant fits to the data points at $r_{\text {out }} \geq 10$ are shown with the dotted lines.

since the present image bin size is larger than the extent of the PSF, the convolution with the PSF is not important. Also, the $\beta$-model function varies slowly within the $5^{\prime \prime}$ bins in most cases, so integration within the image bin can be replaced by the value at the center of the bin. We confirmed these with simulations by comparing two cases with and without convolution in the fitting model. As shown in Fig. 7, we find the difference of the two cases to be negligibly small as long as the core radius is larger than the bin size, $5^{\prime \prime}$. We also confirmed that both cases well reproduce the assumed $\beta$ value of 0.6 within reasonable statistical errors. Thus in order to make the computation time shorter, we skip the convolution with the PSF and the integration over the bin. If the best-fit value (and the error domain) of the core radius is smaller than the bin size, we regard it as an upper limit.

In Fig. 5, we showed a $\chi^{2}$ contour map on the $\beta-r_{\mathrm{c}}$ plane where the other free parameters, $S_{0}$ and $C$, are optimized at each point of the plane. The two parameters are strongly coupled and the allowed parameter range exists in an elongated region. We quote the $90 \%$ confidence intervals on the best-fit parameters. Table 2 lists the results of single $\beta$-model fitting. For \#43 A1758 and \#79 MS1054.5-0321, because the fitting parameters did not converge properly, we optimized the parameters within the range of $\beta \leq 3$.

We compared the results of $\beta$-model fitting with Hashimotodani (1999) for 27 ROSAT/HRI clusters with $0.1<$ $z<0.78$ and Ettori et al. (2004) for 10 high redshift Chandra clusters with constrained model parameters. We found that there is a good agreement for $\beta$ and $r_{\mathrm{c}}$ measurements between our and Hashimotodani samples within their statistical errors. Furthermore for 8 of the 10 high redshift clusters, there is a good agreement between our and the Ettori et al. samples within the $90 \%$ statistical errors. For the two highest redshift samples, \#70 RX J1347.5-1145 $(z=0.451)$ and \#71 3C 295 $(z=0.4641), r_{\mathrm{c}}$ is systematically smaller by about $40 \%$ compared to the Chandra measurements. $\beta$ is also systematically

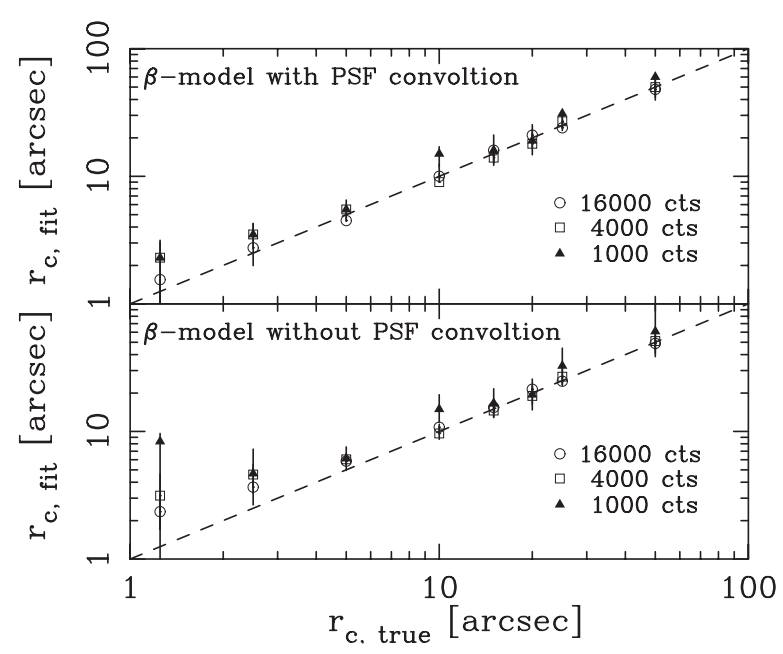

Fig. 7. Reproducibility of $r_{\mathrm{c}}$ in the single $\beta$-model analysis. The $x$-axis is the assumed core radius in the simulation cluster image, and the $y$-axis is the core radius derived from fitting with two different models: the $\beta$-model with and without the PSF convolution. The results for three different photon counts are shown in each panel.

smaller with the mean ratio of 0.93 for 10 high redshift samples, although the difference is the $\sim 2 \sigma$ effect. We consider that the limited sensitivity of the HRI for the outer part of the cluster emission may cause the underestimation of $\beta$ for the highest redshift $(z>0.4)$ samples.

As a result, the fractions of clusters with $\chi^{2}$ values exceeding the $90 \%$ and $99 \%$ confidence levels are 25/79 $(=0.32)$ and $11 / 79(=0.14)$, respectively. The fractions are larger than expected only by the statistical errors. Thus there may be some systematic errors that are not well explained by the single $\beta$-model. For some clusters, systematic deviations from the current model are particularly seen in the central regions (e.g. \#13 A1689), which will be discussed in the next subsection.

We also evaluated an X-ray significance radius, $r_{\mathrm{X}}$, representing the extension of the observed cluster X-ray emission at which the best-fit $\beta$-model surface brightness becomes equal to the $3 \sigma$ background level. The results are also listed in Table 5 .

\subsection{Radial profile fitting with a double $\beta$-model}

For some clusters, systematic residuals are seen in the results of single $\beta$-model fitting. As often seen in nearby clusters, this may be attributed to the presence of central excess emission. The excess component is often represented by an additional $\beta$-model component (Jones \& Forman 1984), whose core radius is $10 \sim 200 h_{50}^{-1} \mathrm{kpc}$, and on average $60 h_{50}^{-1} \mathrm{kpc}(\mathrm{Mohr}$ et al. 1999). This corresponds to only several bins of the present analysis even at $z=0.1$. It is not easy to constrain such central emission in the distant clusters. To evaluate the statistical significance of the central emission, we attempted two methods. We restrict this analysis to the 45 regular clusters because the irregular clusters are often accompanied by substructures, which can cause artificial double structures in their radial profiles.

In the first analysis, we exclude central bins from the fit and investigate the variation of single $\beta$-model parameters against 


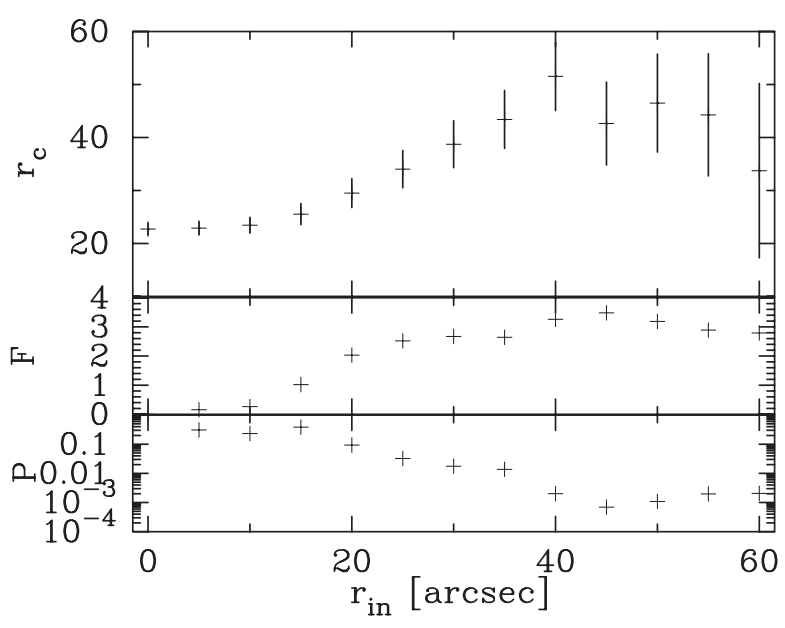

Fig. 8. Effect of the inner cutoff radius in the single $\beta$ model fitting. The results for \#13 A1689 are shown as an example. From top to bottom, the resultant values of $r_{\mathrm{c}}$ [arcsec], $F$ (see Sect. 3.5 for definition) and the probability of exceeding the $F$ value, $P$, are shown.

the inner cutoff radius, $r_{\text {in }}$. We test the improvement in the $\chi^{2}$ value of the fit with the F-test compared to the case of $r_{\text {in }}=0$ (Fig. 8). We define the value of $F$ as $F=\left(\left(\chi_{1}^{2}-\chi_{2}^{2}\right) /\left(v_{1}-\right.\right.$ $\left.\left.v_{2}\right)\right) /\left(\chi_{2}^{2} / v_{2}\right)$, where $\chi^{2}$ and $v$ are the minimum $\chi^{2}$ value and the degrees of freedom, and the suffix " 1 " and " 2 " correspond to the case of $r_{\text {in }}=0$ and $r_{\text {in }}=n$ pixels, respectively $\left(v_{1}-v_{2}=n\right)$. For nine clusters, we find that the probability of exceeding the $F$ value, $P$, rises at a certain $r_{\text {in }}$. For seven such cases, the core radius also starts increasing at the same inner cutoff radius. This indicates that the nine clusters have significant two core sizes. Moreover for the seven of the nine the core radius of the inner component at which the significant improvement of $\chi^{2}$ starts is roughly $r_{\mathrm{c}}$.

In the second analysis, we assumed the double $\beta$-model composed of different core radii $\left(r_{1}<r_{2}\right)$,

$S(r)=\sum_{i=1}^{2} S_{i}\left[1+\left(\frac{r}{r_{i}}\right)^{2}\right]^{-3 \beta_{i}+1 / 2}+C$,

and performed fitting to the radial profiles (Fig. 4b). Because the inner slope $\beta_{1}$ is insensitive to the fit, we linked it to the outer value, $\beta_{1}=\beta_{2}$. We tested the statistical significance of the improvement of the $\chi^{2}$ value by the F-test against the single $\beta$-model; $F=\left(\left(\chi_{\mathrm{s}}^{2}-\chi_{\mathrm{d}}^{2}\right) /\left(v_{\mathrm{s}}-v_{\mathrm{d}}\right)\right) /\left(\chi_{\mathrm{d}}^{2} / \nu_{\mathrm{d}}\right)$, where the suffixes "s" and "d" stand for the case of the single $\beta$-model and the double $\beta$-model, respectively. We found that nine of the regular clusters have significance above the $95 \%$ level. Those nine clusters are the same clusters for which the double core nature is found in the previous analysis. We refer to the nine clusters with significant double structure as "double- $\beta$ " clusters and show the result of the double $\beta$-model fitting in Table 3 .

We have plotted the two core radii against the core radius derived by the single $\beta$-model for the nine "double- $\beta$ " clusters in Fig. 9a. It is remarkable that one of the two cores is nearly consistent with the core of the single $\beta$-model, namely $r_{\mathrm{c}} \sim r_{1}$ or $r_{\mathrm{c}} \sim r_{2}$. This indicates that the single $\beta$-model tends (a)
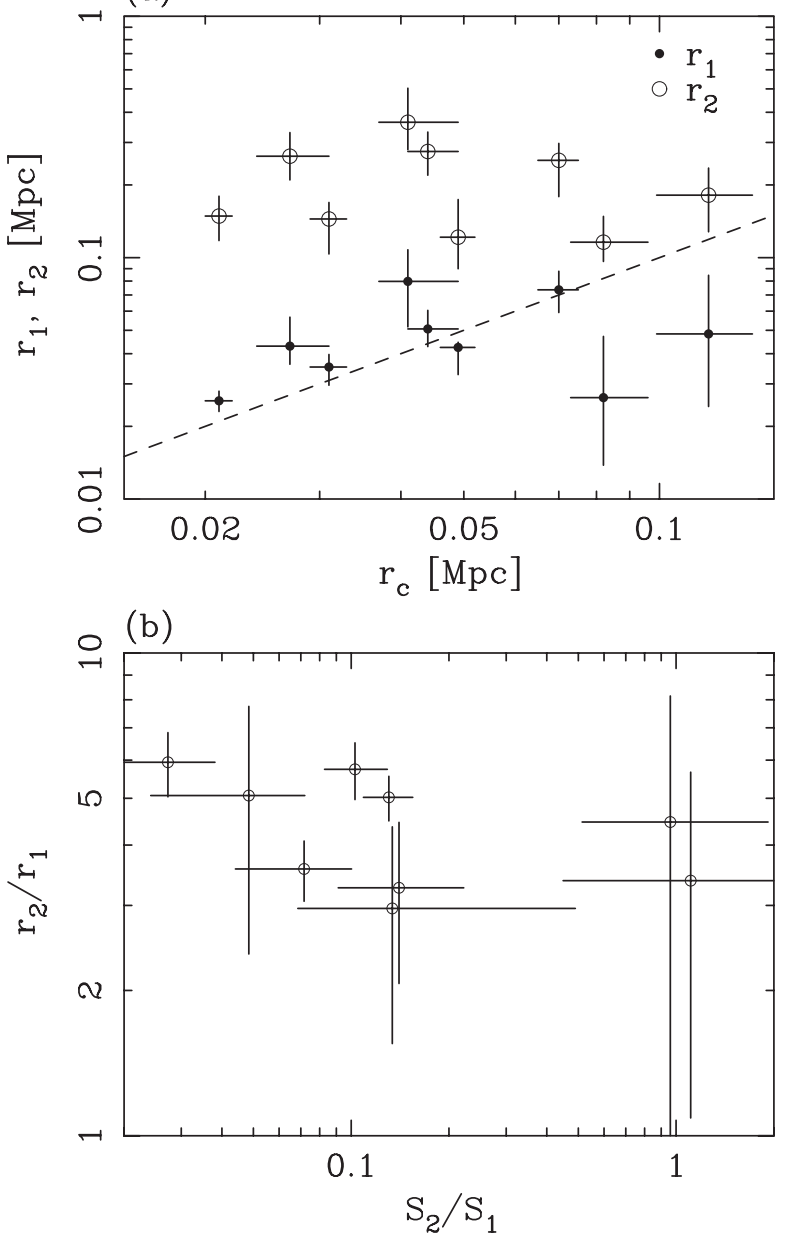

Fig. 9. Core radii derived by the double $\beta$-model for the nine clusters with significant double structure. The panel a) shows the relation between two core radii of the double $\beta$-model and that of the single $\beta$-model, where the inner and outer cores of the double $\beta$ model are shown with the filled and open circles, respectively. The panel b) shows the ratio of the two cores and the ratio of the two normalization factors in Eq. (2). The two data points whose $S_{2} / S_{1}$ are large $(\sim 1)$ in the panel b) correspond to the two clusters with large core radius of the single $\beta$-model in the panel a).

to detect the one of the two components that is more dominant. We also show the ratio of two cores versus the ratio of two normalization factors in Fig. 9 b. $r_{2} / r_{1}$ is $\sim 4$ on average, while $S_{2} / S_{1}$ ranges from 0.01 to 1 . These are consistent with nearby clusters (Mohr et al. 1999). We can classify them into two groups: inner core dominated clusters $\left(S_{2} / S_{1} \lesssim 0.1\right)$ and outer core dominated clusters $\left(S_{2} / S_{1} \sim 1\right)$. For the inner core dominant clusters, the single $\beta$-model fit picks up the inner $\beta$-model component, while for the outer core dominant clusters, the outer component is picked up. In Table 2 the inner/outer core dominant clusters are denoted with $\mathrm{W}(1) / \mathrm{W}(2)$.

We then investigated the reproducibility of the best-fit parameters of the double $\beta$-model utilizing Monte-Carlo simulations. We made ten simulation clusters for each set of model parameters described below and fitted them with the double $\beta$-model. We assumed $r_{1}=3$ pixels $\left(=15^{\prime \prime}\right)$ as a typical core 
radius of the inner component and several different values of $r_{2}$ between 6 and 24 pixels. We fixed $\beta_{1}=\beta_{2}=0.67$. For the intensity ratio, we checked two typical cases, i.e. $S_{2} / S_{1}=0.1$ and 1.0. We then generated 75000 events within a radius of $12^{\prime}$, which are typical total counts for double- $\beta$ clusters including background. We confirmed that the two core radii are well determined as long as $r_{2} / r_{1} \geq 3$. Thus, at least for the nine double- $\beta$ clusters we detected, we can conclude that the model parameters are well-determined by the model fitting.

\subsection{Fraction of double $\beta$ clusters}

We also find that the double- $\beta$ clusters are found only at $z<0.3$ and the ratio to the regular clusters at $z<0.3$ is $32 \%$. However we have to take into account the fact that such double structures are difficult to find at higher $(z>0.3)$ redshifts because of the low surface brightness. In order to constrain the double structure at higher redshifts, we estimated the upper limits of the possible additional $\beta$-model component. For this purpose we fitted the radial profile with double $\beta$-model with the ratio of the two cores fixed at the average of nine double- $\beta$ clusters, $r_{2} / r_{1}=4$. We need to consider two cases: (1) the inner component is dominant, namely the inner core radius is approximately the core radius of the single $\beta$-model, $r_{1} \sim r_{\mathrm{c}}$, and (2) the outer component is dominant, $r_{2} \sim r_{\mathrm{c}}$. We thus performed the fit with $r_{1} \sim r_{\mathrm{c}}$ or $r_{2} \sim r_{\mathrm{c}}$ as the initial value. For some cases, the fit converged to certain best-fit values or upper limits of the fitting parameters. However in some cases the fit did not converge. In such cases we fixed $r_{1}$ or $r_{2}$ at the value of $r_{\mathrm{c}}$ obtained from the single $\beta$-model fitting.

We find that in addition to the nine double- $\beta$ clusters, there are several other clusters that may contain a second component. In case (1) the outer component is marginally detected for seven clusters at the $90 \%$ confidence level. For other 17 clusters, the upper limit of the surface brightness of the outer component is within the range $(0.01-0.1) \mathrm{S}_{1}$ which is comparable to the range for the seven inner-core dominant double- $\beta$ clusters. In case (2) the inner component is marginally detected for three clusters, and the upper limit is consistent with the two double- $\beta$ clusters for the other 16 . In Table 2 the clusters with marginal inner/outer component are denoted with $\mathrm{S}(1) / \mathrm{S}(2)$.

We finally obtain the fraction of clusters with marginal double- $\beta$ structure at $z>0.3$ to be $35 \%$. It is comparable to the occurrence of the double structures in the lower redshift systems within the Poisson errors. Thus we cannot conclude that there is significant evolution of the fraction of the double- $\beta$ clusters in the regular clusters within the observed redshift range. On the other hand, Ettori et al. (2004) noted based on the $\beta$-model analysis that their high redshift $(z>0.4)$ samples do not show any significant double structure in the surface brightness distribution. Thus there might be a trend of evolution in the core structure of the ICM distribution starting around $z \sim 0.4$. We suggest that this should be confirmed by further observations.

\section{Spectral analysis}

\subsection{Data reduction}

We retrieved the ASCA data sets from the High Energy Astrophysics Science Archive Research Center (HEASARC) at NASA/Goddard Space Flight Center and the DARTS Online Service at the Institute of Space and Astronautical Science (ISAS) that were screened with the standard REV-2 processing. We use the FTOOLS analysis package to reduce the cluster spectra, and calculate the response functions of the telescopes and the detectors.

The GIS was operated in the PH-nominal mode during observations. The SIS has several choices between the CCD data modes (FAINT or BRIGHT) and the CCD clocking modes (1CCD or 2CCD or 4CCD). For observations done in a mixture of the FAINT and BRIGHT modes, we can combine converted BRIGHT (on-board FAINT) and on-board BRIGHT mode data.

First we extacted the X-ray images in the $0.7-10 \mathrm{keV}$ for the GIS and the $0.5-10 \mathrm{keV}$ for the SIS, respectively. The two sensors in the same system, namely GIS-2, -3 and SIS-0, -1 are added together. We fitted the projected images to Gaussian functions and determined the peak positions. We accumulate spectra from a circular region centered on the Gaussian peak, where the extraction radii are $6^{\prime}$ and $3^{\prime}$ for the GIS and the SIS, respectively. We will discuss the effect of the contamination from foreground/background point sources in the next subsection. We select a larger integration area for the GIS because the FWHM of the point spread function of the GIS detector alone depends on the incident photon energy $E \mathrm{keV}$, and is given by $0.5 \times(5.9 / E)$ (The ASCA Data Reduction Guide 2002). The FWHM for soft photons is wider than for hard photons; the smaller integration radius would make the spectrum harder, resulting in a systematically higher temperature. This is a serious effect for a distant cluster with apparent core size larger than $1^{\prime}$ when the GIS integration radius is smaller than $\sim 4^{\prime}$. Note that $6^{\prime}$ corresponds to $\sim 1 \mathrm{Mpc}$ at $z=0.1$, and it mostly covers the cluster region corresponding to $\Delta_{\mathrm{c}}=500$ (see Sect. 5.2.1 for definition). The systematic error in estimating the bolometric luminosity due to the fixed integration radii will be discussed in the next subsection.

We subtract background spectra that were obtained during blank-sky observations. Because the ASCA background has a detector position dependency, we extract them from the same region as the cluster in the detector coordinates.

The instrument response can be split into two parts: a redistribution matrix (RMF), which specifies the channel probability distribution for a photon of given energy, and an effective area curve (ARF), which specifies the telescope area and window absorption. We utilized the latest version of the GIS RMFs, gis2v4_0.rmf and gis3v4_0.rmf, while we generate the SIS RMF using the FTOOL sisrmg. We built the ARF files with the ASCAARF program appropriate for the cluster extended emission, summing the ARFs for each bin in the cluster image region according to the weight of the photon counts. 


\subsection{Fitting with Raymond-Smith model}

In order to measure the average, emission-weighted X-ray temperature of the gas, we fitted the SIS and GIS spectra simultaneously with a thin-thermal plasma emission model from Raymond \& Smith (1977). There are four parameters in the spectral model, the temperature $k T$, the metallicity relative to the solar abundance $Z$, the redshift $z$, and the normalization factor. The redshift of each object was fixed at the cataloged value in the NASA/IPAC Extragalactic Database (NED). The fitting function was convolved with the telescope and detector response functions. In the spectral fitting, we used the XSPEC version 9.0 analysis software (Arnaud 1996). We rebinned the spectral channels so that each bin contains at least 40 photons.

Yaqoob (1999) pointed out that fitting with fixed $N_{\mathrm{H}}$ result in a systematically high temperature because of the serious decrease of the low-energy efficiency of the SIS since early 1994. To avoid the problem, the absorption column density $N_{\mathrm{H}}$ was allowed to vary. Note that in the observation of \#57 A402, which was done during the $\mathrm{AO} 7$ phase, a serious reduction in the efficiency below $1 \mathrm{keV}$ was seen in the SIS spectra, so we used only the GIS data for this cluster.

We checked the contribution of foreground/background contaminating sources in the ASCA spectra. We picked up the point sources whose photon counts are greater than $10 \%$ of the cluster from the HRI source lists. Excluding regions of $r=1^{\prime}$ circles around the sources, we recalculate the spectra, responses and backgrounds to determine the temperature by the Raymond-Smith model fitting. Note that Energy Encircled Function at $1^{\prime}$ is approximately $\sim 0.3$ for the GIS and $\sim 0.4$ for the SIS, respectively. We estimated the difference of the best-fit temperatures with and without point sources excluded relative to the $1 \sigma$ error of the measurement, and found that the contribution of the point-like sources does not affect the temperature measurement for most cases, except for \#56 CL0500-24, \#66 CL0024+17, \#76 3C 220.1, and A963. For \#20 A963, we excluded one nearby source from the spectral region of the GIS and the SIS. More specific analyses are described in Ota et al. (1998) for CL0500-24, Soucail et al. (2000) for CL0024+17, Ota et al. (2000) for 3C 220.1. In the case of CL0500-24 and CL0024+17, only the SIS data were used. Table 4 lists the results of the spectral analysis.

We compared our results with values published in White (2000) (there are 26 clusters in common) to find a good agreement between the two measurements with a mean temperature ratio of 1.04. We also compared 6 high redshift clusters with constrained ASCA temperature with Ettori et al. (2004). The temperatures for 5 clusters are statistically consistent between the two results, while there is systematic difference for \#71 3C 295. As for 3C 295, Ettori et al. (2004) excluded the central emission associated with the AGN, thus our temperature measurement may be affected by the AGN.

We estimated the X-ray luminosity in the $2-10 \mathrm{keV}$ band from the GIS flux, $L_{X}\left(6^{\prime}\right)$, and converted it into the bolometric luminosity, $L_{\mathrm{X}, \mathrm{bol}}\left(6^{\prime}\right)$, using the emissivity of the RaymondSmith plasma model. In order to check the systematic error of the luminosity estimation, we calculated the bolometric luminosity by integrating the $\beta$-model surface brightness distribution determined with the ROSAT HRI within the same integration area, to find that two estimations are consistent within about $15 \%$. Then we derived the bolometric luminosity within $r_{500}$ (see Sect. 5.2.1), $L_{\mathrm{X} \text {,bol }}$, by multiplying $L_{\mathrm{X} \text {,bol }}\left(6^{\prime}\right)$ with the ratio of the luminosities within $r_{500}$ to $6^{\prime}$ using the $\beta$-model. In Table 4 we listed $L_{\mathrm{X}}\left(6^{\prime}\right), L_{\mathrm{X}, \text { bol }}\left(6^{\prime}\right)$, and $L_{\mathrm{X}, \mathrm{bol}}$.

\section{Cluster parameters}

In Sect. 3 we analyzed the cluster surface brightness distribution under the assumption that the gas is isothermal, and the spatial distribution is described by the single $\beta$-model or the double $\beta$ model. In Sect. 4 , we determined the average X-ray temperature with the ASCA spectral data. The X-ray images and the fitting results of individual clusters are shown in Fig. C.1. We will derive some physical quantities of the clusters from the X-ray parameters obtained from the analysis and investigate the redshift dependence of these parameters and the histograms.

\subsection{Parameters from spectral analysis}

\subsubsection{Redshift dependence of spectral parameters}

We show the spectral parameters obtained with the RaymondSmith fittings as a function of redshift in Fig. 10. In the plot of the temperature, we quoted the results of nearby clusters (Mohr et al. 1999) for comparison. There is no significant change in the temperature over a wide redshift range, particularly in $z<0.5$. On the other hand, some clusters with $z>0.5$ resulted in very high temperatures, though their errors are not well-constrained due to the limited photon statistics and the error range overlaps with the high temperature clusters in $z<0.5$.

As indicated from Fig. 10a, it is hard to detect a cluster whose X-ray flux is below $\sim 10^{-13} \mathrm{erg} \mathrm{s}^{-1} \mathrm{~cm}^{-2}$ due to the detection limit of $A S C A$. This flux corresponds to $\sim 1 \times 10^{44} \mathrm{erg} \mathrm{s}^{-1}$ at $z=0.5$ and to $k T \sim 2.5 \mathrm{keV}$ from the $L_{\mathrm{X}}-T$ relation. Thus at $z>0.5$, clusters with temperature lower than $2.5 \mathrm{keV}$ are barely detected. We consider that this can account for the higher average temperature at $z>0.5$.

\subsubsection{Histograms of the spectral parameters}

In Fig. 11 we plot the number of occurrence of each spectral parameter to study how the samples are distributed in the parameter space, regardless of the redshift. We show the distributions of the regular and the irregular clusters separately as well as the distributions of all the clusters. As a result, there is no clear difference between the distributions of the regular and irregular clusters. In Table 8 we summarize the mean and the standard deviation of the spectral parameters.

\subsection{Parameters from $\beta$-model analysis}

\subsubsection{Definitions of cluster parameters}

From the $\beta$-model fits and spectral analysis we obtained four primary X-ray parameters $\left(k T, \beta, r_{\mathrm{c}}, S_{0}\right)$ to describe the 

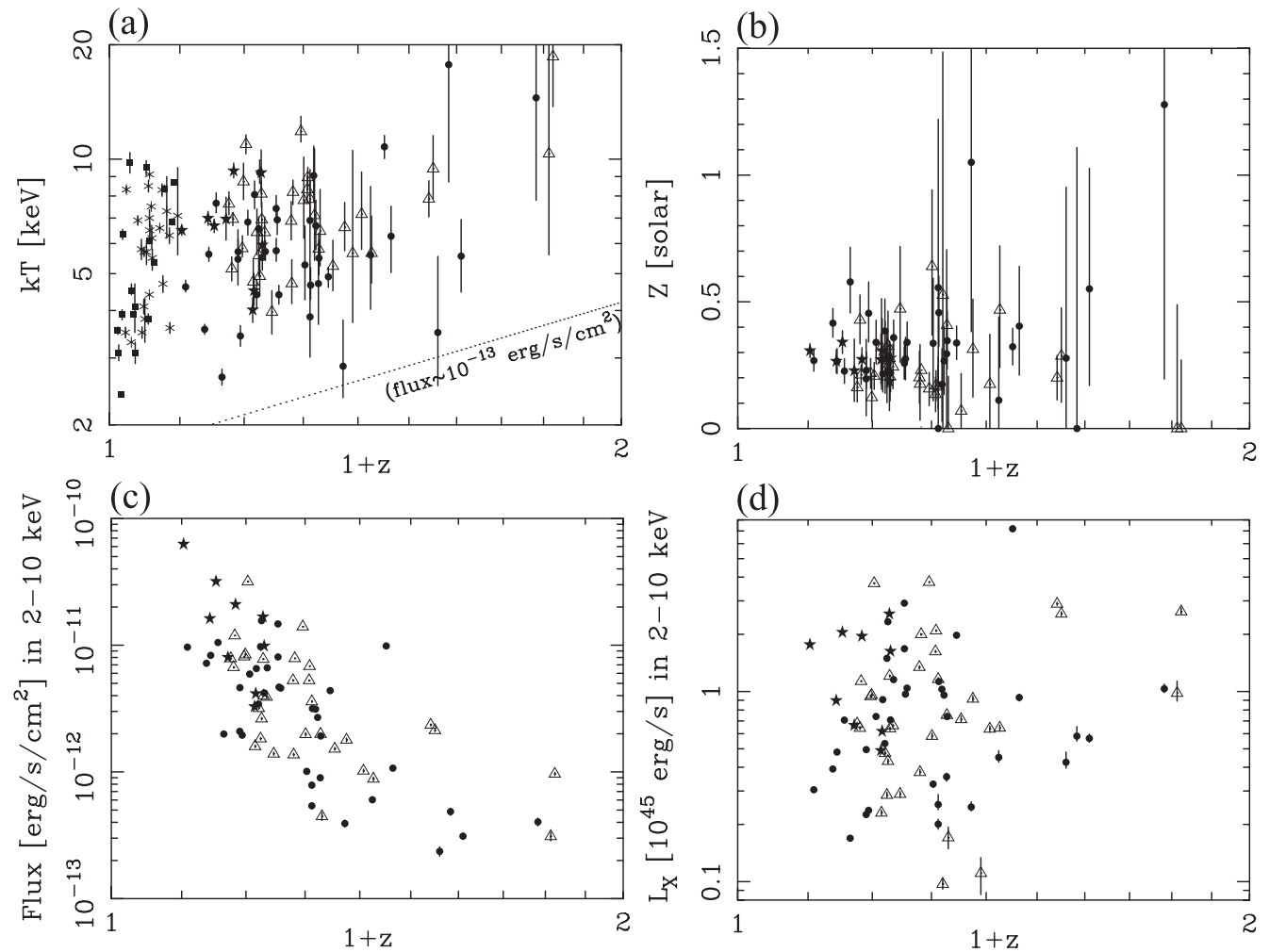

Fig. 10. X-ray temperature a), metal abundance b), the $2-10 \mathrm{keV}$ flux c) and the $2-10 \mathrm{keV}$ luminosity within $r<6^{\prime}$ d) measured with ASCA. At $z>0.1$, the filled circles and the open triangles are the regular and the irregular clusters in our sample. The filled stars show the double- $\beta$ clusters in our sample. The ASCA sensitivity curve is indicated with the dotted line in the panel of $k T$, assuming the luminosity-temperature relation. The temperatures of clusters with $z<0.1$ were taken from Mohr et al. (1999). The asterisks and the filled squares denote the single- $\beta$ and double- $\beta$ clusters in the nearby sample, respectively.

Table 8. Means and standard deviations of cluster parameters.

\begin{tabular}{lllllll}
\hline \hline Parameter & \multicolumn{2}{c}{ Regular (45) } & \multicolumn{2}{c}{ Irregular (34) } & \multicolumn{2}{c}{ All (79) } \\
\cline { 2 - 6 } & Mean & Standard deviation & Mean & Standard deviation & Mean & Standard deviation \\
\hline$k T[\mathrm{keV}]$ & 6.3 & 2.8 & 7.2 & 2.7 & 6.8 & 2.8 \\
$Z$ [solar $]$ & 0.33 & 0.22 & 0.24 & 0.15 & 0.29 & 0.19 \\
$L_{\mathrm{X}, \mathrm{bol}}[\mathrm{erg} / \mathrm{s}]$ & $1.7 \times 10^{45}$ & $1.9 \times 10^{45}$ & $1.7 \times 10^{45}$ & $1.7 \times 10^{45}$ & $1.7 \times 10^{45}$ & $1.8 \times 10^{45}$ \\
$\beta$ & 0.56 & 0.11 & 0.73 & 0.61 & 0.64 & 0.32 \\
$r_{\mathrm{c}}\left[h_{70}^{-1} \mathrm{Mpc}\right]$ & 0.076 & 0.060 & 0.273 & 0.259 & 0.163 & 0.202 \\
$n_{\mathrm{e} 0}\left[h_{70}^{1 / 2} \mathrm{~cm}^{-3}\right]$ & $3.6 \times 10^{-2}$ & $2.9 \times 10^{-2}$ & $7.4 \times 10^{-3}$ & $8.4 \times 10^{-3}$ & $2.4 \times 10^{-2}$ & $2.6 \times 10^{-2}$ \\
$\rho_{0}\left[h_{70}^{2} \mathrm{~g} \mathrm{~cm}^{-3}\right]$ & $2.5 \times 10^{-24}$ & $2.5 \times 10^{-24}$ & $7.3 \times 10^{-25}$ & $2.7 \times 10^{-24}$ & $1.7 \times 10^{-24}$ & $2.7 \times 10^{-24}$ \\
$t_{\text {cool }}[\mathrm{Gyr}]$ & 4.4 & 4.6 & 15.6 & 8.1 & 9.4 & 8.5 \\
$r_{500}\left[h_{70}^{-1} \mathrm{Mpc}^{-1}\right.$ & 0.96 & 0.22 & 1.1 & 0.4 & 1.0 & 0.3 \\
$M_{500}\left[h_{70}^{-1} M_{\odot}\right]$ & $4.3 \times 10^{14}$ & $5.1 \times 10^{14}$ & $9.7 \times 10^{14}$ & $2.4 \times 10^{15}$ & $6.7 \times 10^{14}$ & $1.6 \times 10^{15}$ \\
$M_{\mathrm{gas}}\left[h_{70}^{-5 / 2} M_{\odot}\right]$ & $7.1 \times 10^{13}$ & $4.3 \times 10^{13}$ & $9.5 \times 10^{13}$ & $6.5 \times 10^{13}$ & $8.2 \times 10^{13}$ & $5.5 \times 10^{13}$ \\
$f_{\mathrm{gas}}\left[h_{70}^{-3 / 2}\right]$ & 0.20 & 0.08 & 0.18 & 0.07 & 0.20 & 0.07 \\
\hline
\end{tabular}

intracluster gas. From these parameters, we evaluate several important quantities that characterize properties of the clusters and the ICM. Below we summarize the definitions of these quantities.

1. Electron density at the cluster center: $n_{\mathrm{e} 0}$

The central electron density can be determined from the central surface brightness $S_{0}, \beta, r_{\mathrm{c}}$ and the temperature. However, from the present analysis, we obtained the central surface photon flux convolved with the telescope and the detector responses. In this case $S_{0}$ can be written as

$$
\begin{aligned}
S_{\mathrm{p} 0}\left(E_{1}, E_{2}\right)= & \int_{E_{1}}^{E_{2}} \mathrm{~d} E^{\prime} \int \mathrm{d} E R\left(E^{\prime}, E\right) A(E) n_{\mathrm{e} 0} n_{\mathrm{H} 0} \\
& \times \frac{\Lambda_{\mathrm{p}}(T, Z, E, z) \sqrt{\pi} r_{\mathrm{c}}}{4 \pi\left(D_{\mathrm{A}}(1+z)\right)^{2}} \frac{\Gamma(3 \beta-1 / 2)}{\Gamma(3 \beta)},
\end{aligned}
$$

where $S_{\mathrm{p} 0}\left(E_{1}, E_{2}\right)$ [counts s $\mathrm{cm}^{-2}$ ] is the photon flux in the $E_{1}-E_{2}$ energy band, $R\left(E^{\prime}, E\right)$ and $A(E)$ 

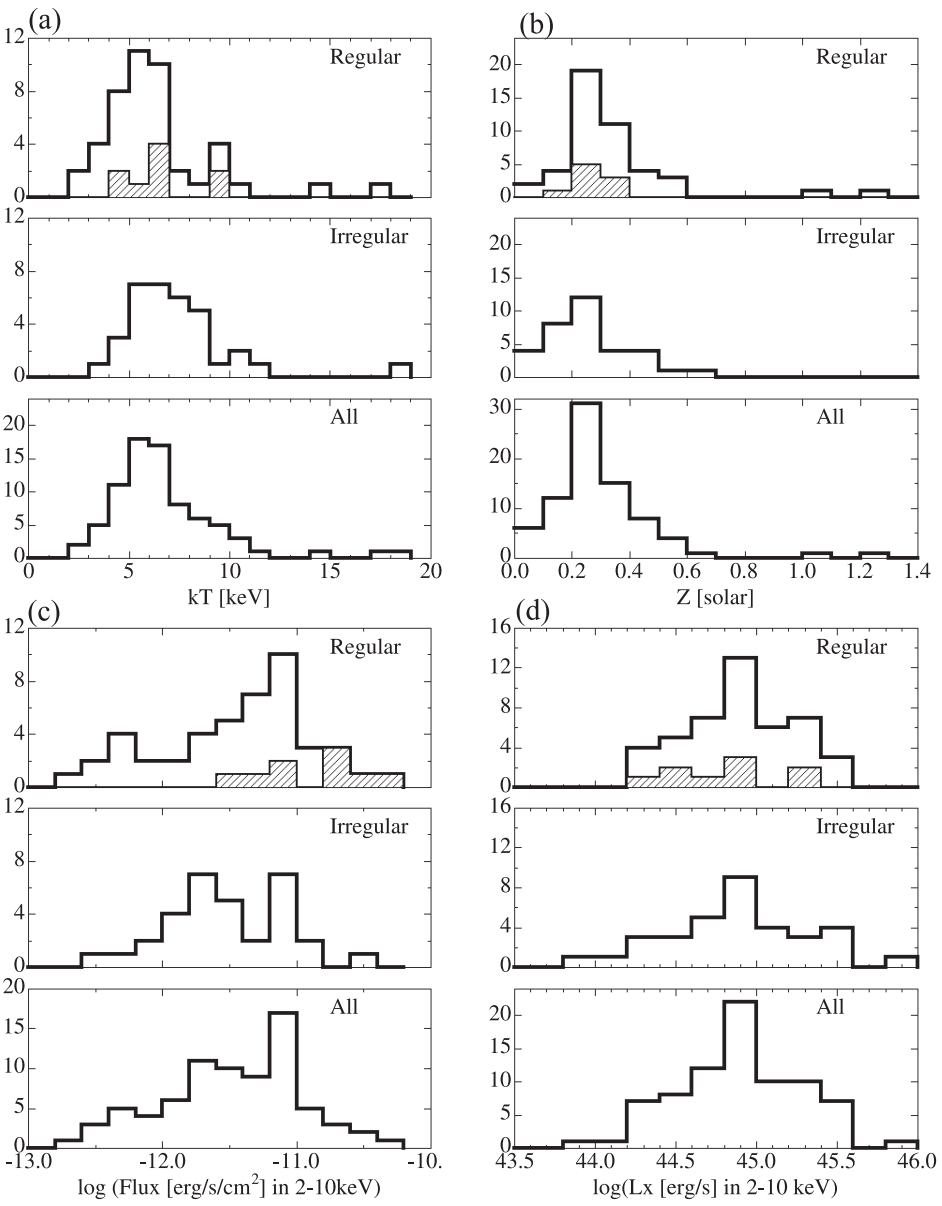

Fig. 11. Histograms of the spectral parameters determined with ASCA for 79 clusters (open). The panels a) and b) show the best-fit temperature and the metal abundance determined from the Raymond-Smith model fitting, respectively. The X-ray flux and the luminosity within $r<6^{\prime}$ in the 2-10 keV band estimated with the GIS are shown in c) and d), respectively. The results of the double- $\beta$ clusters are superposed (hatched).

respectively represent the response function of the detector and the effective area of the X-ray telescope/detector system, $\Lambda_{\mathrm{p}}(T, Z, E, z)$ the emissivity in units of photons s $\mathrm{sm}^{-1} \mathrm{keV}^{-1}$ for the object at redshift $z$. $D_{\mathrm{A}}$ is the angular size distance to the cluster. We utilized the XSPEC program to perform the convolutions with the detector response functions. We adopt $n_{\mathrm{H} 0}=\left(\mu_{\mathrm{e}} / \mu_{\mathrm{H}}\right) n_{\mathrm{e} 0}$, where $\mu_{\mathrm{H}}=1.40$ and $\mu_{\mathrm{e}}=1.167$.

2. Cluster mass profile and density profile: $M(r), \bar{\rho}(r), \rho_{0}$ Assuming that the intracluster gas is in hydrostatic equilibrium, the following condition is satisfied:

$\frac{k T}{\mu m_{\mathrm{p}}}\left(\frac{\mathrm{d} \ln \rho_{\mathrm{gas}}}{\mathrm{d} \ln r}+\frac{\mathrm{d} \ln T}{\mathrm{~d} \ln r}\right)=-\frac{G M(r)}{r}$.

In the case that the cluster gas is isothermal and has a $\beta$ model density profile, $\rho_{\text {gas }}(r)=\rho_{\text {gas }}(0)\left(1+\left(r / r_{\mathrm{c}}\right)^{2}\right)^{-3 \beta / 2}$, where $\rho_{\text {gas }}(0)=\mu_{\mathrm{e}} m_{\mathrm{p}} n_{\mathrm{e} 0}$, the total mass contained within the radius $r$, is estimated from

$M(r)=\frac{3 k T \beta r}{\mu m_{\mathrm{p}} G} \frac{\left(r / r_{\mathrm{c}}\right)^{2}}{1+\left(r / r_{\mathrm{c}}\right)^{2}}$.

The average density within $r$ is then

$\bar{\rho}(r)=\frac{M(r)}{\frac{4}{3} \pi r^{3}}=\frac{\rho_{0}}{1+\left(r / r_{\mathrm{c}}\right)^{2}}$, where $\rho_{0} \equiv 9 k T \beta / 4 \pi G \mu m_{\mathrm{p}} r_{\mathrm{c}}^{2}$ is the central total matter density.

3. Cooling timescale of the gas at the cluster center: $t_{\text {cool }}$. We estimate the radiative cooling timescale of the intracluster gas at the cluster center as

$t_{\mathrm{cool}}=\frac{3 k \sqrt{T}}{q_{\mathrm{ff}} n_{\mathrm{e} 0}}$,

where $q_{\mathrm{ff}}$ is related to the volume emissivity of thermal Bremsstrahlung through $\epsilon_{\mathrm{ff}}=q_{\mathrm{ff}} n_{\mathrm{e}}^{2} T^{1 / 2}$.

4. Cluster limiting radius (overdensity radius) and cluster mass: $r_{500}$ and $M_{500}$.

We determine a cluster limiting radius within which the average density $\bar{\rho}(r)$ is equal to $\Delta_{\mathrm{c}}$ times the critical density of the universe at the collapse time; namely

$\bar{\rho}(r)=\Delta_{\mathrm{c}} \rho_{\text {crit }}\left(z_{\mathrm{col}}\right)$.

We adopt a fixed overdensity of $\Delta_{c}=500$, which is justified in a sense that Evrard et al. (1996) suggested from their numerical simulations to use this value to study the gas properties and that the hydrostatic assumption is not valid beyond this radius, and that Finoguenov et al. (2001) showed that an assumption of isothermality also works at such overdensities. Since we do not know the redshift of the cluster 
(a)

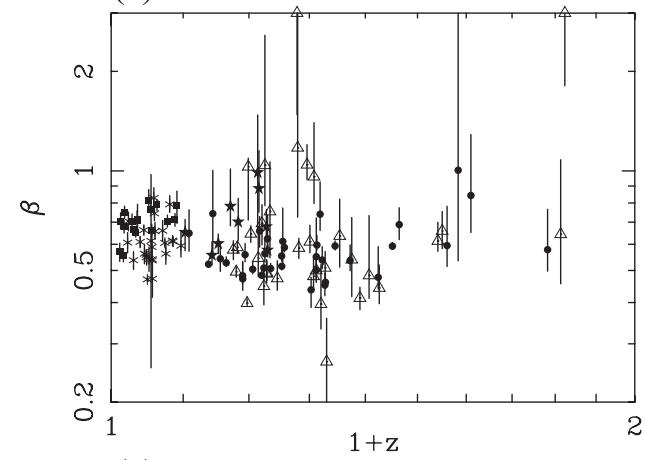

(c)
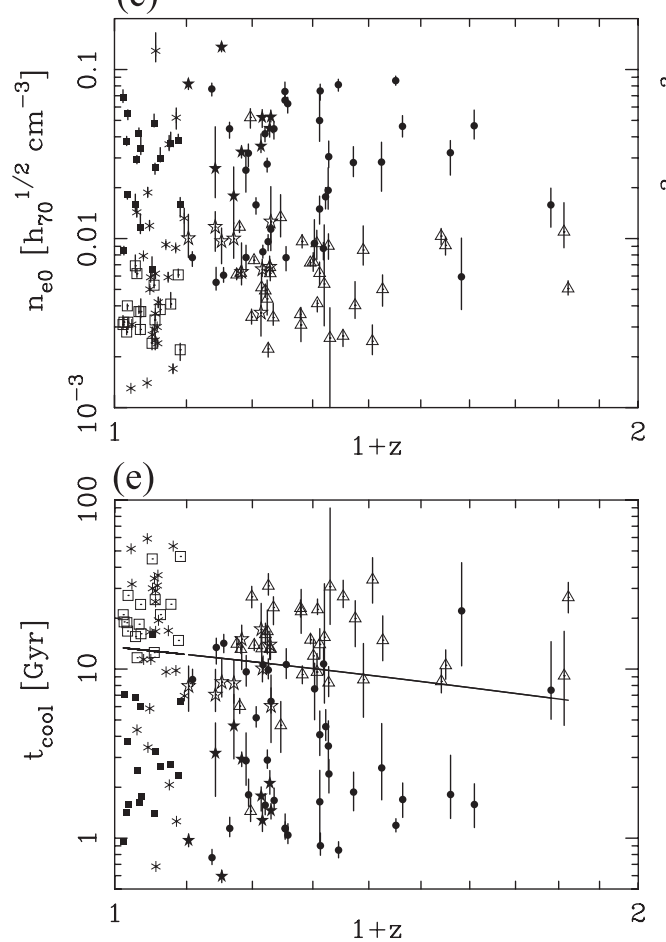

(b)

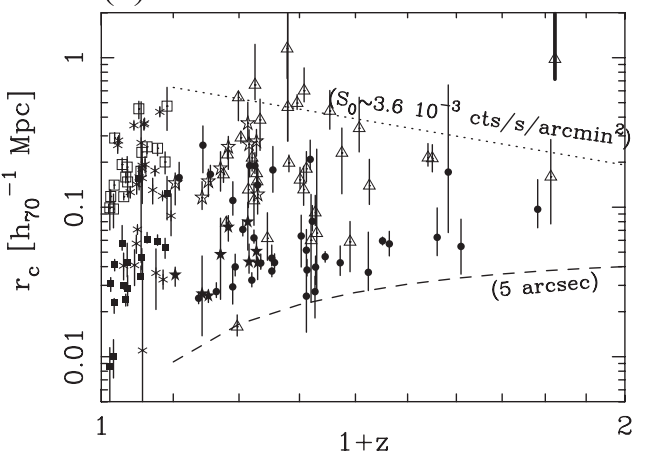

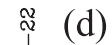

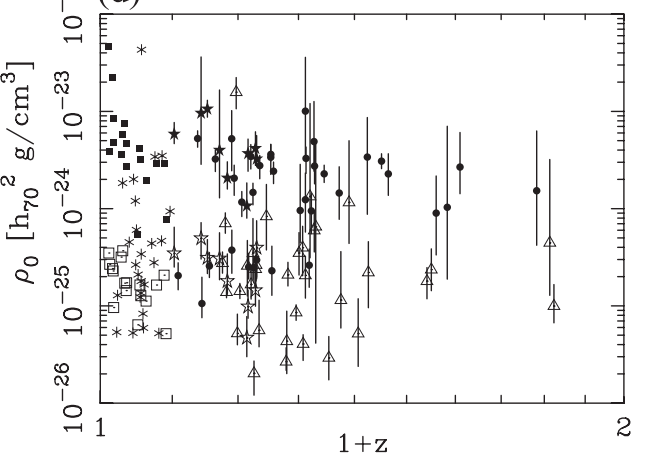

Fig. 12. Results from $\beta$-model analysis. In the panels a)-e), $\beta, r_{\mathrm{c}}, n_{\mathrm{e}}, \rho_{0}$, and $t_{\text {cool }}$ are shown. At $z>0.1$, the filled circles and the open triangles are the regular and the irregular clusters in our sample. The filled stars and the open stars show the inner core and the outer core of the double- $\beta$ clusters in our sample. At $z<0.1$ the filled squares and the open squares denote the inner and outer components of the double- $\beta$ clusters in the nearby Mohr et al. (1999) sample. The asterisks denote the nearby single- $\beta$ clusters. In the panel of $r_{\mathrm{c}}$, the selection effects due to the sensitivity and the spatial resolution of the ROSAT HRI are indicated with the dotted line and the dashed line, respectively. In the panel of $t_{\text {cool }}$ we show a curve on which $t_{\text {cool }}$ is equal to the age of the Universe at the cluster redshift.

collapse, the most simple assumption is that the clusters are observed just after they are formed, i.e. $z_{\mathrm{col}}=z_{\mathrm{obs}}$. We will determine the overdensity radius, $r_{500}$ under this assumption and calculate the hydrostatic mass within $r_{500}, M_{500}$, from Eq. (5).

5. Gas mass, and gas-mass fraction within $r_{500}: M_{\mathrm{gas}}$ and $f_{\mathrm{gas}}$. The gas mass within $r_{500}$ is derived with

$$
\begin{aligned}
M_{\text {gas }} & =\int_{0}^{r_{500}} \rho_{\text {gas }}(r) 4 \pi r^{2} \mathrm{~d} r \\
& =4 \pi \rho_{\text {gas }}(0) r_{\mathrm{c}}{ }^{3} \int_{0}^{x_{500}}\left(1+x^{2}\right)^{-3 \beta / 2} x^{2} \mathrm{~d} x,
\end{aligned}
$$

where $x=r / r_{\mathrm{c}}$ and $x_{500}=r_{500} / r_{\mathrm{c}}$. Then we obtain the gas-mass fraction with $f_{\text {gas }}=M_{\text {gas }} / M_{500}$.
Among the four X-ray parameters, the temperature $k T$ is independently determined from the other three $\beta$-model parameters. However, the statistical errors of the three parameters are coupled to one another. In particular the coupling between $\beta$ and $r_{\mathrm{c}}$ is strong (Fig. 5). We determined the statistical errors of the cluster parameters listed above with this coupling taken into account. For that purpose we first determined the error domain, i.e. the statistically allowed parameter region, in the four dimensional parameter space. Then, evaluating the cluster parameters for all combinations of the X-ray parameters in the domain, we determined the maximum and the minimum parameter values of the domain. For the double- $\beta$ clusters, we also calculated those cluster parameters from the double $\beta$-model. The methods of calculation are similar to those shown above. The details are shown in Appendix A. 
(a)

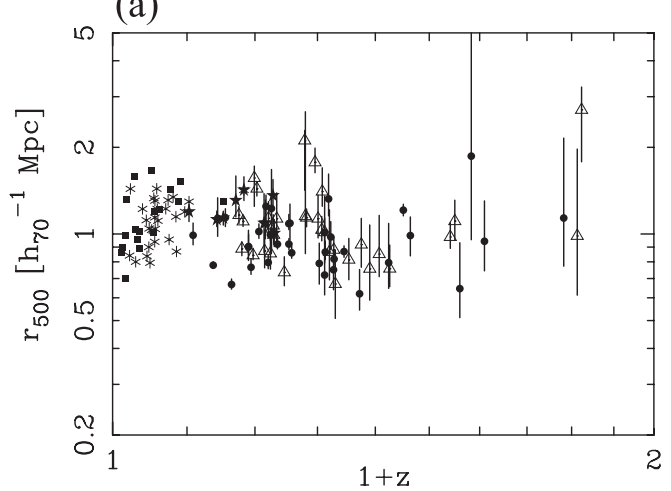

(c)

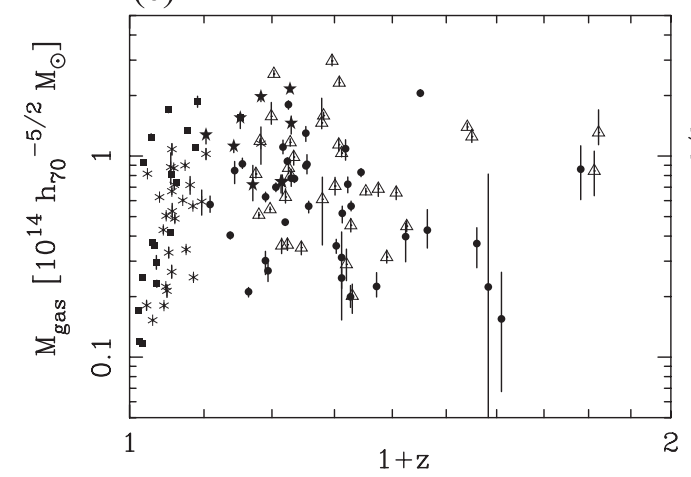

(b)

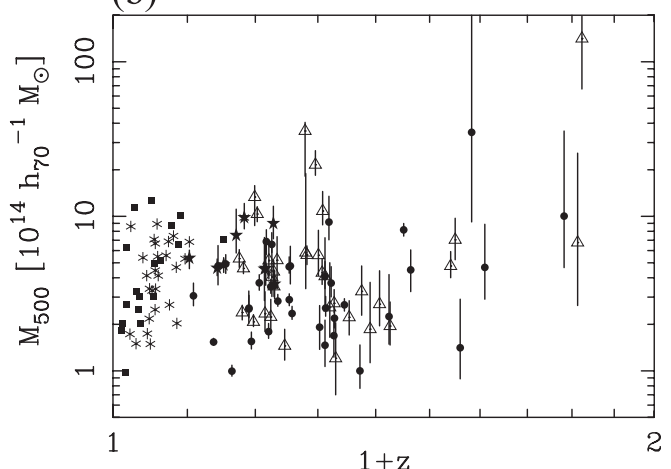

(d)

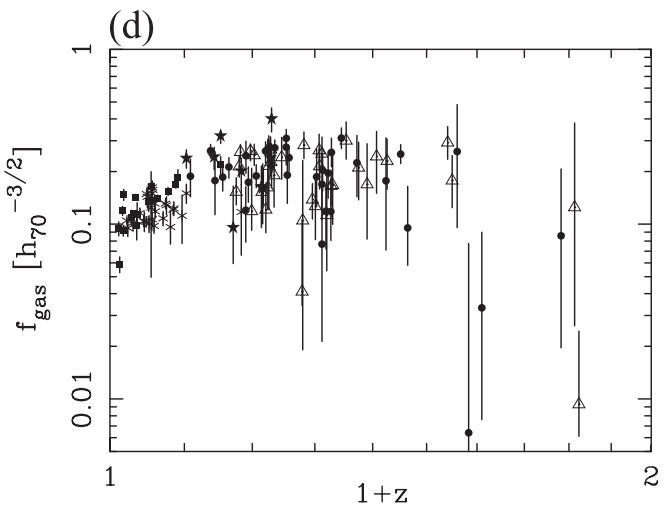

Fig. 13. $r_{500}, M_{500}, M_{\text {gas }}$, and $f_{\text {gas }}$ derived from $\beta$-model analysis are shown in the panels a)-d). See Sect. 5.2.1 for definitions of the parameters. The meanings of the symbols are the same as in Fig. 12 .

\subsubsection{Redshift dependence of X-ray parameters}

We plot the $\beta$-model parameters, and the parameters derived from those X-ray parameters as functions of redshift in Fig. 12. We show the results of double- $\beta$ model fits, and their inner and outer components are distinguished by different symbols. In the figures we also plotted the parameters taken from Mohr et al. (1999) for clusters with $z<0.1$.

We do not see in those figures any clear redshift dependence in the distributions of the X-ray parameters except for the parameters related to $r_{500}$ shown in Fig. 13. We will go back to these parameters in Sect. 5.2.3 and focus on the parameters that do not involve the overdensity radius.

From Fig. 12b, we notice that the core radius shows an apparent redshift dependence. As noted in Ota \& Mitsuda (2002), the core radius shows a remarkably large cluster-to-cluster dispersion, spanning over two orders of magnitude. The core radii of the irregular clusters are systematically larger than those of the regular clusters, and there seems to be a gap in the $r_{\mathrm{c}}$ distribution at around $0.1 h_{70}^{-1} \mathrm{Mpc}$. The regular clusters also show a similar bimodal distribution in $r_{\mathrm{c}}$ but the fraction of the larger $r_{\mathrm{c}}$ group decreases with increasing $z$.

To investigate the selection effect, we created simulation clusters with the Monte-Carlo method and performed the analysis on the simulation clusters. We scale the count rate of MS0906.5+1110 at $z=0.18$ to estimate the expected total counts for a $40 \mathrm{ksec}$ observation of a cluster at $z=0.5$ with a typical luminosity $L_{\mathrm{X}} \sim 1 \times 10^{45} \mathrm{erg} \mathrm{s}^{-1}$. The expected cluster counts are about 600 counts. We simulated a series of cluster images with various core sizes and found that the signal-to-noise ratio is quite low for clusters with large cores of $r_{\mathrm{c}} \gtrsim 400 \mathrm{kpc}$ at $z>0.5$. Based on the results we estimated the sensitivity of the current HRI observation to be $S_{0} \sim 3.6 \times 10^{-3}$ counts s ${ }^{-1} \operatorname{arcmin}^{-2}$. We show the sensitivity curve in Fig. 12b. Thus the redshift dependencies of the core radius can be explained by a selection effect.

Therefore we conclude that the $\mathrm{X}$-ray parameters, temperature, core radius, $\beta$, and the central electron density are consistent with showing no significant trend of evolution at $z \lessgtr 0.5$.

\subsubsection{Gas-mass fraction within $r_{500}$ and the systematic error}

Although the X-ray parameters that are directly determined from observation do not show strong redshift-dependence, we find a weak redshift dependence in the overdensity radius, which approximately follows $r_{500} \propto\left(1+z_{\text {obs }}\right)^{-0.6}$ (Fig. 13a). The dependence is likely to be introduced when we define $r_{500}$ by Eq. (8), i.e. $\bar{\rho}(r)=\Delta_{\mathrm{c}} \rho_{\mathrm{c}}\left(z_{\mathrm{obs}}\right)$. In other words, the redshift dependency is introduced by the assumption, $z_{\text {col }}=z_{\text {obs }}$. We confirmed that such a negative dependence disappears when we assume a constant $z_{\text {col }}$ independently of $z_{\text {obs }}$, for example $z_{\mathrm{col}}=1$.

In Fig. 13d, we show the gas-mass fraction inside $r_{500}$ determined with $z_{\mathrm{col}}=z_{\mathrm{obs}}$. We do not see a clear dependence on the redshift in our own data. We then obtain the average gasmass fraction of our sample of 79 distant clusters to be

$\left\langle f_{\text {gas }}\right\rangle=(0.20 \pm 0.07) h_{70}^{-3 / 2}$ 
where the quoted error is the standard deviation of the clusterto-cluster variation. On the other hand the average gas-mass fraction of the nearby samples of Mohr et al. (1999) is $\left\langle f_{\text {gas }}\right\rangle_{\text {nearby }}=0.12 \pm 0.03$ (note we recalculated the value with Eq. (8) and $\Delta_{\mathrm{c}}=500$ under the assumption of $z_{\mathrm{col}}=z_{\mathrm{obs}}$ ). Mohr et al. (1999) estimated their systematic errors to be $\sim 10 \%$. Although our distant sample seems to show higher $f_{\text {gas }}$ values in comparison to the nearby samples, the two results are consistent with each other within their errors. If we further compare our result with the baryon density in the Universe determined by the Wilkinson Microwave Anisotropy Probe (Spergel et al. 2003), $\Omega_{\mathrm{b}} / \Omega_{0}=0.16$, it is again higher although they are in agreement within the errors. We will thus examine the possible systematic errors of $f_{\text {gas }}$ estimation due to (1) the choice of limiting radius; (2) the calibrations of the X-ray telescope/detector systems; (3) the assumption of $z_{\mathrm{col}}=z_{\mathrm{obs}}$; and (4) the effect of the temperature gradient.

(1) We have defined the cluster limiting radius with $\Delta_{\mathrm{c}}=500$ and the resultant value is typically $r_{500} \sim 1 \mathrm{Mpc}$ for the current sample. On the other hand, the extent of the observed X-ray emission, $r_{\mathrm{X}}$, is found to be larger for most of the clusters (Fig. C.1) and $r_{\mathrm{X}} / r_{500}=1.5$ on average. Thus we do not need to worry about the effect of extrapolation in the current $f_{\text {gas }}$ estimation. The average gas-mass fraction within $r_{\mathrm{X}}$ is derived as $(0.24 \pm 0.06) h_{70}^{-3 / 2}$, which agrees with Eq. (10) within the errors.

(2) Since the determination of the gas-mass fraction requires absolute calibrations of the X-ray telescope/detector systems of ROSAT and ASCA, we carefully examined the calibrational errors and found that they can cause at maximum $25 \%$ errors in $f_{\text {gas }}$ (see Appendix B for details).

(3) The assumption of $z_{\mathrm{col}}=z_{\mathrm{obs}}$ may be a source of uncertainty in $f_{\text {gas }}$. We can infer $z_{\text {col }}$ from the condition that the central mass density should be higher than the average mass density, namely $\rho_{0}>\bar{\rho}\left(r_{500}\right)$. Since the observed range of $\rho_{0}$ is $\sim 2 \times 10^{-26}-1 \times 10^{-23} \mathrm{~g} \mathrm{~cm}^{-3}$, we obtain $z_{\mathrm{col}} \lesssim 1.3-18$, where the smaller (larger) value corresponds to the clusters with large (small) core radii. Thus it is likely that the clusters with large $r_{\mathrm{c}}$ were formed at $z_{\text {col }} \lesssim 1.3$.

We then vary the formation redshift, $z_{\mathrm{col}}$ for which we calculate the critical density, $\rho_{\text {crit }}\left(z_{\text {col }}\right)$ and find the radius where the measured matter density is 500 times $\rho_{\text {crit }}\left(z_{\text {col }}\right)$. If we simply assume a fixed formation redshift for all the clusters, ranging from 0.5 to 1.5 , and calculate the mean gas-mass fractions within $r_{500}$ in the same manner as Eq. (10), $\left\langle f_{\text {gas }}\right\rangle$ varies from 0.18 to 0.12 with a typical standard error of 0.05 . Thus the $f_{\text {gas }}$ estimation largely depends on the assumption of $z_{\mathrm{col}}$. However such an effect is expected to be more serious for the low-redshift clusters, whose average gas-mass fraction was measured to be $\left\langle f_{\text {gas }}\right\rangle_{\text {nearby }} \sim 0.12$ (Mohr et al. 1999; Sanderson et al. 2003). Thus we may not attribute the systematic error in measuring $f_{\text {gas }}$ for the distant sample to the assumption of $z_{\mathrm{col}}$.

(4) The emission-weighted temperature reflects the temperature of the cluster core region. Then, if there is a significant temperature drop at the center, it may cause an overestimation of the gas-mass fraction because the cluster hydrostatic mass estimation is more sensitive to the temperature profile than the gas mass. Such temperature drops were usually found in cluster cores with short ( $\sim$ a few Gyr) cooling timescales. The spectral analysis of the cooling flow clusters with the XMM-Newton and the Chandra satellites showed that the temperature drops typically by a factor of 3 over the central $r \lesssim 200 \mathrm{kpc}$ region (e.g. Tamura et al. 2001; Schmidt et al. 2001). We then estimated the emission-weighted temperature within $1.5 \mathrm{Mpc}$ (corresponding to the typical integration radius for the GIS spectra) assuming the radial temperature profile of $T(r) \propto r^{0.2}$ (from Fig. 1 of Tamura et al. 2001) and the $\beta$-model surface brightness distribution with $r_{\mathrm{c}}=50 \mathrm{kpc}$ and $\beta=2 / 3$ to find that it is lower by about $30 \%$ than that of the outer $(r>0.1 \mathrm{Mpc})$ region. On the other hand, we obtained the mean gas-mass fraction for the 26 regular clusters with $t_{\text {cool }} \leq 3$ Gyr to be $\left\langle f_{\text {gas }}\right\rangle=0.22 \pm 0.08$, which is larger by $20 \%$ than that of the rest of the sample. Thereby we consider that, for at most $1 / 3$ of the samples, $f_{\text {gas }}$ may be overestimated to some extent, depending on the degree of the temperature gradient. However, considering the fact that the correlation between $f_{\text {gas }}$ and $t_{\text {cool }}$ is weak as well as that the ranges of $f_{\text {gas }}$ for clusters with short and long cooling timescales are not very different, the effect of the temperature gradient in estimating the mean gas-mass fraction for all the samples is suggested to be not large compared to the clustercluster variation. For more accurate measurements of $f_{\text {gas }}$, we need to constrain the temperature profiles for the individual clusters and reduce the measurement uncertainties.

\subsubsection{Histograms of the X-ray parameters}

Since we found no significant evolution in the X-ray parameters, we will investigate the distribution of X-ray parameters obtained from the $\beta$-model analysis without distinguishing the clusters by redshift. In Fig. 14, we show the histograms of the $\mathrm{X}$-ray parameters. We find that both $k T$ and $\beta$ are distributed in ranges smaller than 1 order of magnitude, while $r_{\mathrm{c}}$ and $n_{\mathrm{e} 0}$ are distributed over almost two orders of magnitude.

While the mean value of $r_{\mathrm{c}}$ for all the clusters is $\left\langle r_{\mathrm{c}}\right\rangle=$ $0.163 h_{70}^{-1} \mathrm{Mpc}$, if we treat the regular and the irregular clusters separately, we obtain $\left\langle r_{\mathrm{c}}\right\rangle=0.076 h_{70}^{-1} \mathrm{Mpc}$ and $\left\langle r_{\mathrm{c}}\right\rangle=$ $0.273 h_{70}^{-1} \mathrm{Mpc}$ for the regular and the irregular clusters (see Table 8 ). Thus these are different by a factor of 3 . We also notice that the distributions of the irregular and regular clusters are not separated. Instead, the distribution of the regular clusters has a double-peaked structure, whose core radii corresponding to the two peaks are $50 h_{70}^{-1} \mathrm{kpc}$ and $200 h_{70}^{-1} \mathrm{kpc}$ respectively. Thus there is about a factor of four difference. The peak of the larger core radius coincides with that of the irregular clusters. One may consider that the regular clusters with a large core radius were classified as regular because the counting statistics were not very good. However, we find that the statistics of irregular and regular clusters with a large core are not very different. Thus it is difficult to explain this coincidence just by statistics. The core radius distribution when the nearby Mohr et al. (1999) samples and the our distant samples were added together was shown in Ota \& Mitsuda (2002).

In Fig. 14b, we also show the distributions of the core radii of the double- $\beta$ clusters. We notice that the double-peaked 
(a)
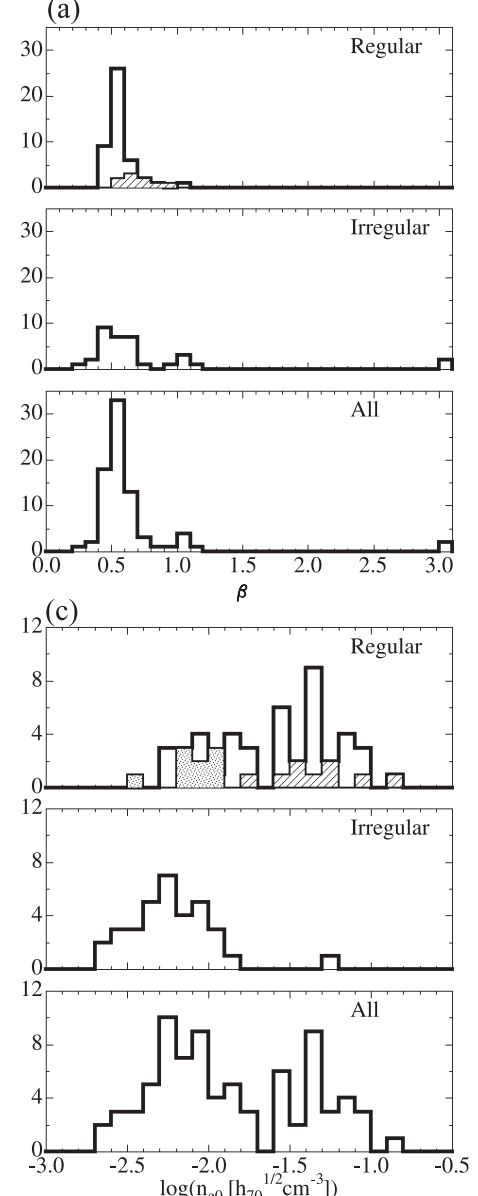

(e)
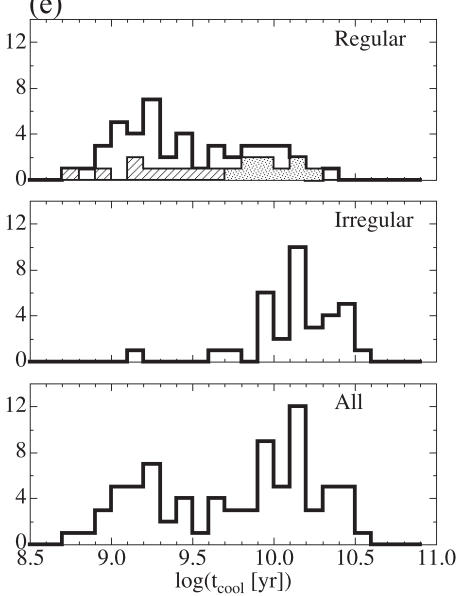
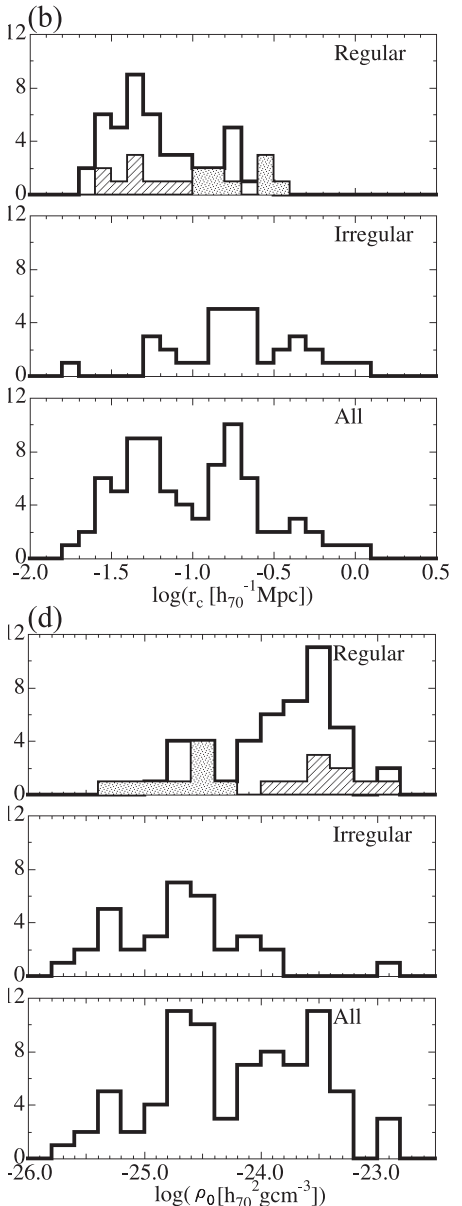

Fig. 14. Histograms of the $\mathrm{X}$-ray parameters determined with the single $\beta$-model for 79 clusters (open). The results of the double $\beta$-model fittings for the nine double- $\beta$ clusters are superposed in the panels, where the hatched and filled regions show contributions of the inner core and the outer core, respectively.

structure of the core radius of the regular clusters seems related to the double $-\beta$ structure because the core radius of the smaller-core component is distributed around the lower peak of the regular-cluster core radius distribution, while the largercore is around the higher peak.

In Sect. 3.5, we have shown that about $20 \%$ of regular clusters have significant double- $\beta$ structures, and for about $60 \%$ of regular clusters, the existence of a similar structure cannot be rejected. We have also shown that there are inner-core dominant and outer-core dominant cases and that a single $\beta$ model fit picks up the core radius of the dominant core (Fig. 9). Thus we consider that the correspondence between the core radius distribution of all the regular clusters and the distribution of double- $\beta$ clusters is not just a coincidence but that it is related to the double core nature of the regular clusters.

The electron density, the dark matter density and the cooling time at the cluster center show similar double-peaked distributions. On the other hand, as shown in Fig. 15, $r_{500}$ and the 

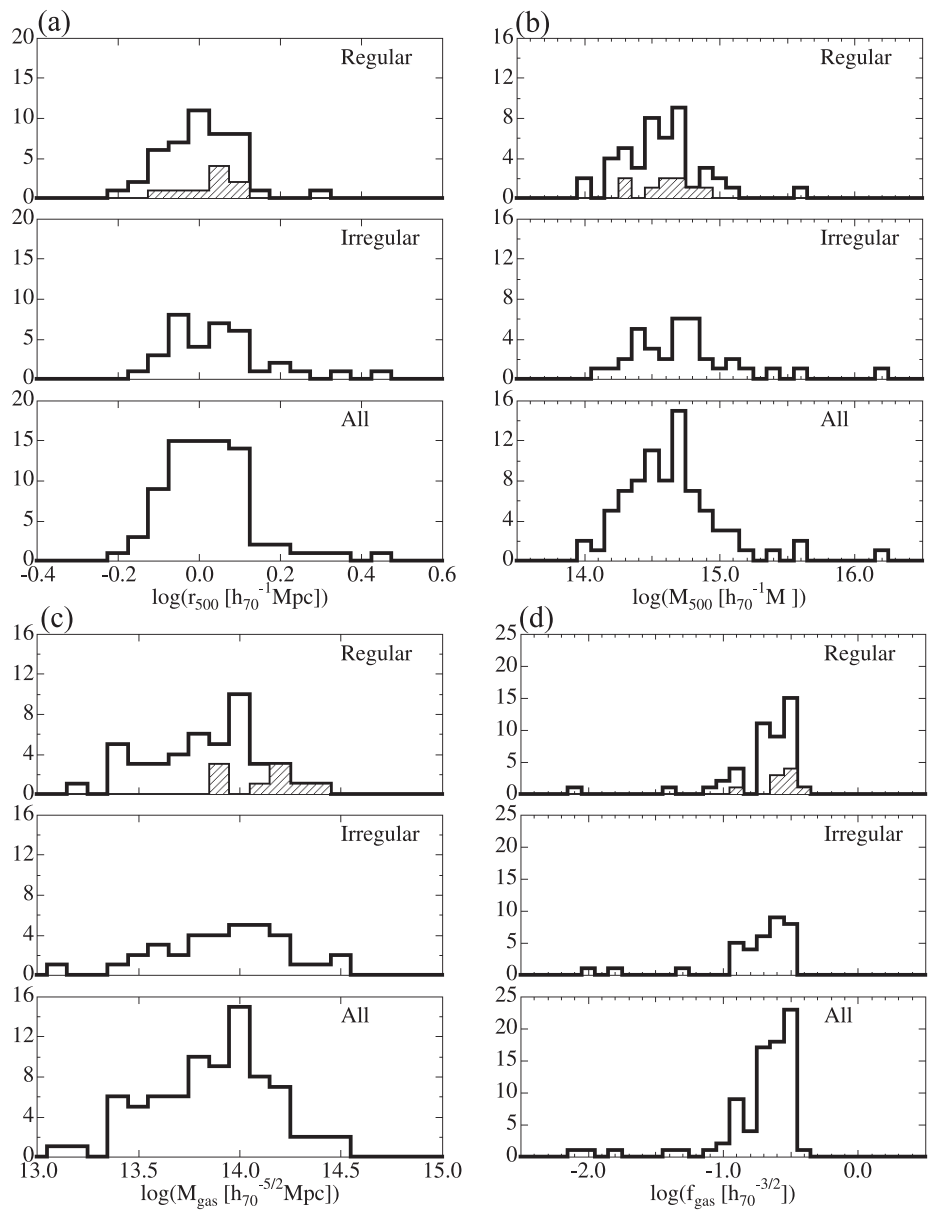

Fig. 15. Histograms of $r_{500}, M_{500}, M_{\text {gas }}$ and $f_{\text {gas }}$ derived with the single $\beta$ model for 79 clusters (open). The results of double $\beta$ clusters are superposed (hatched).

other three parameters evaluated within $r_{500}$ are distributed in ranges smaller than 1 order of magnitude. We will discuss the correlations between the parameters in the next section in more detail. We list the mean and the standard deviation for all the parameters in Table 8 .

\section{Correlations between cluster parameters}

We investigate correlations of various cluster parameters with the X-ray temperature, $k T$ in Sect. 6.1 and also with the core radius, $r_{\mathrm{c}}$ in Sect. 6.2, which showed the distinct double-peaked distribution and thus may provide a clue to understand the structures of clusters. Possible systematic errors will be considered in each subsection.

In the following analysis, we first calculate the Pearson's correlation coefficient, $R_{X Y}=\sum_{i}\left(X_{i}-\bar{X}\right) \sum_{i}\left(Y_{i}-\right.$ $\bar{Y}) / \sqrt{\sum_{i}\left(X_{i}-\bar{X}\right)^{2}} \sqrt{\sum_{i}\left(Y_{i}-\bar{Y}\right)^{2}}$, to measure the strength of the correlation. We take the logarithm of the two measured parameters, $x$ and $y$, namely $X=\log x$ and $Y=\log y$, except for the case of the $Z-T$ and the $\beta-T$ relations. Then if $\left|R_{X Y}\right| \geq 0.3$, we derive the best-fit relation between the two parameters assuming the power-law function. In order to take into account the statistical uncertainties of both the $x$ and $y$ axes, we performed the $\chi^{2}$ minimization in the linear $(Y=a X+b)$ fit by defining $\chi^{2} \equiv \sum_{i}\left(Y_{i}-\left(a X_{i}+b\right)\right)^{2} /\left(a \sigma_{X, i}^{2}+\sigma_{Y, i}^{2}\right)$, where $\sigma_{X, i}$ and $\sigma_{Y, i}$ are the $1 \sigma$ errors for the parameter $X_{i}$ and $Y_{i}$, respectively. Because in all the cases below the fits were not statistically acceptable due to large scatters of the data at the $90 \%$ confidence level, the error ranges of the coefficients $a$ and $b$ were estimated from the dispersions of the data points around the model functions rather than the photon statistics. We excluded two irregular clusters, \#43 A1758 and \#79 MS1054.5-0321, in the analysis except for the $Z-T$ and the $L_{\mathrm{X}, \text { bol }}-T$ relations because their $\beta$-model parameters were not well constrained (Sect. 3.4), although they are plotted in Figs. 16 and 18. We then compare some of the resulting relations with the predictions of the self-similar model.

\subsection{Correlations with the gas temperature}

In Figs. 16a-h, we show eight parameters derived from the spectral and the image analysis, the gas mass, and the gas-mass fraction etc. as a function of X-ray temperature. We mainly show the relations derived for $\Delta_{c}=500$ below. In Table 7, we show the cluster parameters of the individual clusters for the overdensities of $\Delta_{c}=500 \Omega^{0.427}$ and $18 \pi^{2} \Omega^{0.427}$ (Nakamura \& Suto 1997) and in Table 9 the scaling relations with and without consideration of the cosmological factor, $E_{z}=H_{z} / H_{0}$ as noted by Ettori et al. (2004). Since we did not find any strong 
(a)

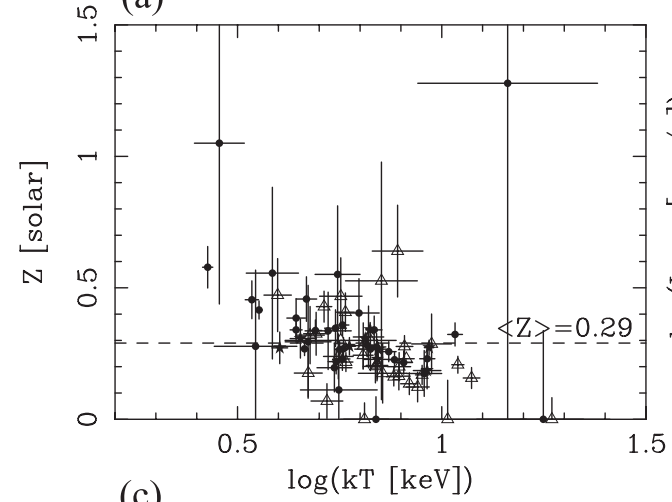

(c)

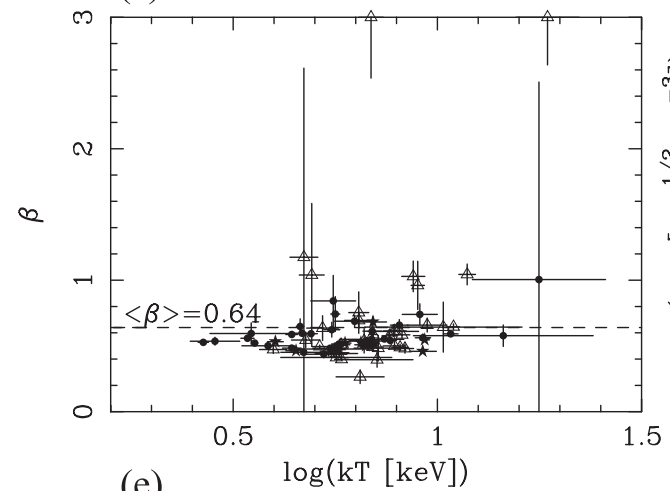

(e)

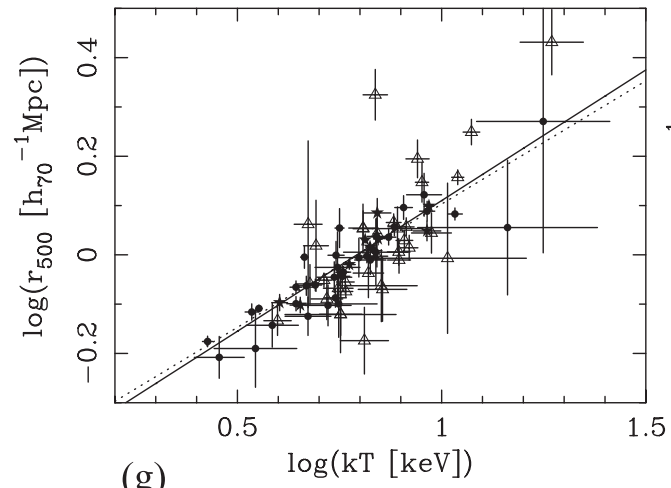

(g)

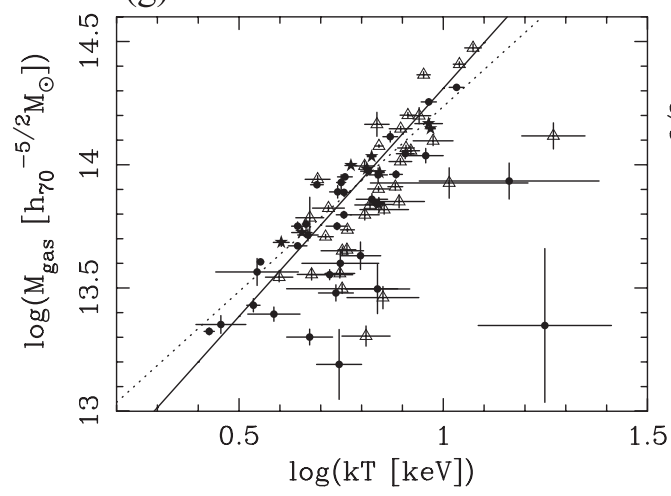

(b)

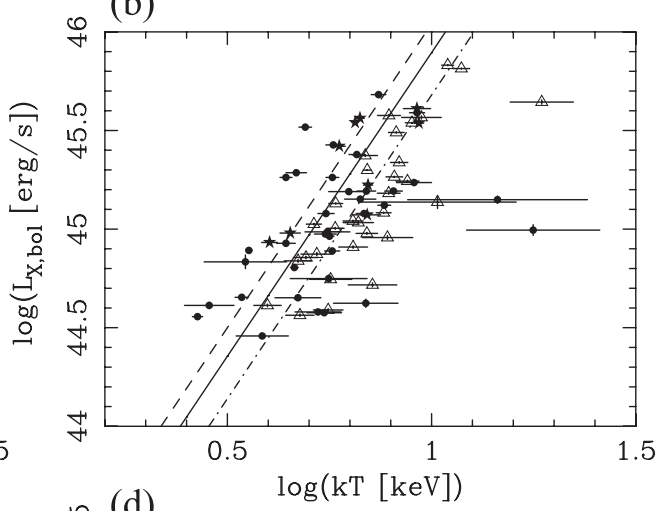

(d)

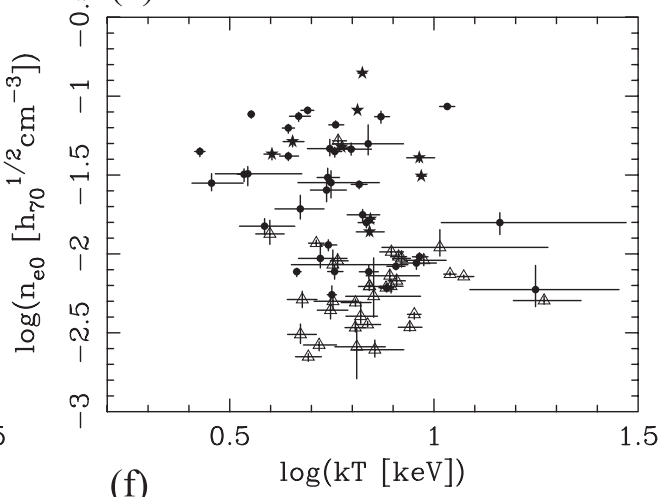

(f)

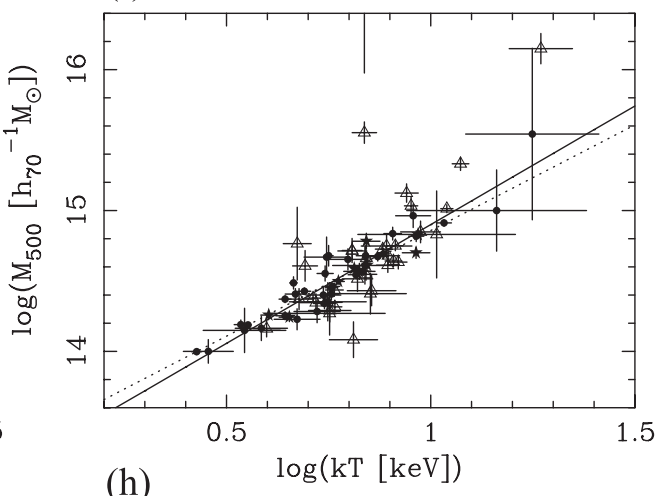

(h)

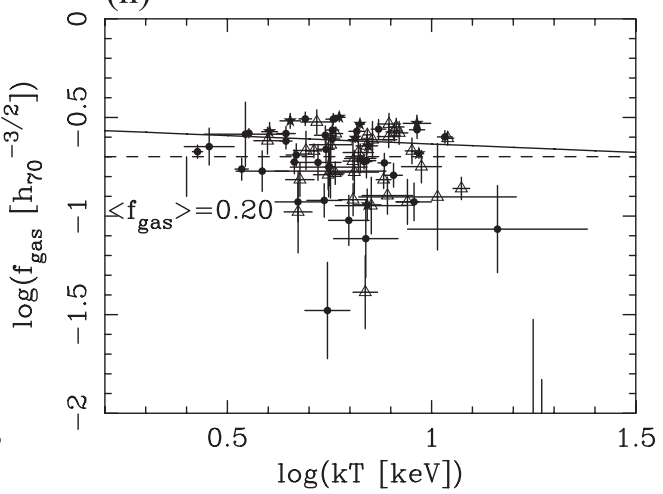

Fig. 16. Relations of the metallicity a), the bolometric luminosity $\mathbf{b}), \beta \mathbf{c}$ ), the central electron density d), the overdensity radius e), the cluster mass $\mathbf{f}$ ), the gas mass $\mathbf{g}$ ), and the gas-mass fraction $\mathbf{h}$ ) with the X-ray temperature. In all the panels the results of the single $\beta$-model fitting are plotted and the single- $\beta$ regular clusters, the single- $\beta$ irregular clusters, and the double- $\beta$ clusters are denoted with the filled circles, the open triangles, and the filled stars respectively. The horizontal and the vertical error bars are $1 \sigma$. In the panels b) and e)-h), the best-fit power-laws for the entire sample are shown with the solid lines. In the panels a), c) and $\mathbf{h}$ ) the sample means are indicated with the dashed lines. In panel $\mathbf{b})$, the best-fit $L-T$ relations for two subgroups with $r_{\mathrm{c}}<0.1 \mathrm{Mpc}$ and $r_{\mathrm{c}}>0.1 \mathrm{Mpc}$ are indicated with the dashed and dash-dotted lines, respectively (see Sect. 6.1 for details). In panels $\mathbf{e}$ )-g), the slopes expected from the self-similar model are shown with the dotted lines. 
Table 9. Scaling relations for the distant clusters.

\begin{tabular}{llll}
\hline \hline \multirow{2}{*}{ Relation } & \multicolumn{3}{c}{$\Omega_{\mathrm{M}}=0.3, \Omega_{\Lambda}=0.7, z_{\mathrm{col}}=z_{\mathrm{obs}}$} \\
\cline { 2 - 4 } & $\Delta_{\mathrm{c}}=500$ & $\Delta_{\mathrm{c}}=500 \Omega^{0.427}$ & $\Delta_{\mathrm{c}}=18 \pi^{2} \Omega^{0.427}$ \\
\hline$L_{\mathrm{X}, \mathrm{bol}}-T$ & $6.53_{-3.82}^{+8.34} \times 10^{42}(k T)^{3.08_{-0.45}^{+0.48}}$ & $7.94_{-4.58}^{+8.92} \times 10^{42}(k T)^{3.01_{-0.42}^{+0.47}}$ & $1.07_{-0.55}^{+1.26} \times 10^{43}(k T)^{2.92_{-0.43}^{+0.40}}$ \\
$M-T$ & $1.64_{-0.26}^{+0.35} \times 10^{13}(k T)^{1.68_{-0.11}^{+0.10}}$ & $2.07_{-0.43}^{+0.35} \times 10^{13}(k T)^{1.68_{-0.08}^{+0.13}}$ & $3.31_{-0.60}^{+0.73} \times 10^{13}(k T)^{1.69_{-0.11}^{+0.11}}$ \\
$M_{\text {gas }}-T$ & $2.88_{-0.64}^{+0.93} \times 10^{12}(k T)^{1.85_{-0.15}^{+0.14}}$ & $4.32_{-0.89}^{+1.28} \times 10^{12}(k T)^{1.80_{-0.15}^{+0.13}}$ & $1.10_{-0.25}^{+0.29} \times 10^{13}(k T)^{1.67_{-0.13}^{+0.15}}$ \\
$f_{\text {gas }}-T$ & $0.28_{-0.06}^{+0.09}(k T)^{-0.08_{-0.14}^{+0.14}}$ & $0.33_{-0.08}^{+0.10}(k T)^{-0.11_{-0.15}^{+0.15}}$ & $0.47_{-0.15}^{+0.16}(k T)^{-0.19_{-0.16}^{+0.20}}$ \\
\hline$E_{z}^{-1} L_{\mathrm{X}, \mathrm{bol}}-T$ & $5.50_{-3.24}^{+7.92} \times 10^{42}(k T)^{3.12_{-0.48}^{+0.51}}$ & $7.24_{-4.04}^{+9.15} \times 10^{42}(k T)^{3.00_{-0.45}^{+0.45}}$ & $1.01_{-0.55}^{+0.99} \times 10^{43}(k T)^{2.89_{-0.39}^{+0.43}}$ \\
$E_{z} M-T$ & $1.73_{-0.32}^{+0.36} \times 10^{13}(k T)^{1.71_{-0.10}^{+0.11}}$ & $2.11_{-0.37}^{+0.45} \times 10^{13}(k T)^{1.73_{-0.11}^{+0.11}}$ & $3.51_{-0.62}^{+0.75} \times 10^{13}(k T)^{1.71_{-0.10}^{+0.11}}$ \\
$E_{z} M_{\text {gas }}-T$ & $3.05_{-0.72}^{+0.94} \times 10^{12}(k T)^{1.88_{-0.15}^{+0.15}}$ & $4.62_{-1.04}^{+1.18} \times 10^{12}(k T)^{1.82_{-0.12}^{+0.15}}$ & $1.19_{-0.28}^{+0.30} \times 10^{13}(k T)^{1.68_{-0.12}^{+0.15}}$ \\
\hline
\end{tabular}

redshift evolution in the observed X-ray properties, we first derive the parameter correlations regardless of their redshifts. We will discuss the correlations in the case where we take into account the sample redshifts later. Note that $k T$ and $r_{\mathrm{c}}$ are in units of $[\mathrm{keV}]$ and $\left[h_{70}^{-1} \mathrm{Mpc}\right]$, respectively.

\section{$-Z-T$}

In Fig. 16a, there seems to be a slight decline of the metallicity, $Z$, against the temperature, however, we found the correlation coefficient, $R_{X Y}$ to be -0.23 for 78 clusters, where \#66 CL0024+17 was not used in the fit because $Z$ was so uncertain that the value was fixed at 0.3 solar in the spectral fitting (Soucail et al. 2000). Thus there is no clear $Z-T$ correlation. This is consistent with the previous results on the metallicity measurements for 21 clusters reported by Mushotzky \& Loewenstein (1997). We thus indicate the mean metallicity of our sample, $\langle Z\rangle=0.29$, with the dotted line in the figure (see also Table 8).

- $L_{\mathrm{X}, \mathrm{bol}}-T$

We find a significant correlation between the bolometric luminosity and the temperature, and $R_{X Y}$ is 0.59 for 77 samples. Note that \#56 CL0500-24 and \#66 CL0024+17 are not included in order to avoid the systematic difference in the integration radius for the spectra because the only SIS spectral data were used for these two clusters due to the serious contamination from the point sources in the vicinity of the clusters (see Sect. 4). We thus obtain the temperatureluminosity relation for the 77 clusters to be

$L_{\mathrm{X}, \mathrm{bol}}\left[\mathrm{erg} \mathrm{s}^{-1}\right]=6.53_{-3.82}^{+8.34} \times 10^{42}(k T)^{3.08_{-0.45}^{+0.48}}$.

$\chi^{2} /$ d.o.f. $=1703 / 75$. As a result, the slope of the relation is consistent with those previously published for the nearby clusters (e.g. Markevitch 1998; Arnaud \& Evrard 1999). However, if compared to the relation for higher redshift clusters by Ettori et al. (2004), they showed a steeper slope of $3.72 \pm 0.47$ ( $1 \sigma$ error).

The large error in the normalization factor in Eq. (11) is affected by the intrinsic scatter around the best-fit relation. We found from Fig. 16b that the normalization factors of the $L_{\mathrm{X}, \mathrm{bol}}-T$ relation are significantly different between the regular and the irregular clusters: for a fixed temperature, the regular clusters tend to have a larger luminosity in comparison to the irregular clusters. The difference becomes more evident if we divide the sample into two subgroups according to the core radius ranges of $r_{\mathrm{c}}<0.1 h_{70}^{-1} \mathrm{Mpc}$ and $r_{\mathrm{c}}>0.1 h_{70}^{-1} \mathrm{Mpc}$ (see also Ota \& Mitsuda 2002). If we fit them separately, fixing the slope at the best-fit value of 3.08 , we obtain

$$
\begin{aligned}
& L_{\mathrm{X}, \text { bol }}\left[\mathrm{erg} \mathrm{s}^{-1}\right]=9.12_{-0.92}^{+1.02} \times 10^{42}(k T)^{3.08} \text { for } r_{\mathrm{c}} \leq 0.1,(12) \\
& L_{\mathrm{X}, \text { bol }}\left[\mathrm{erg} \mathrm{s}^{-1}\right]=3.98_{-0.21}^{+0.22} \times 10^{42}(k T)^{3.08} \text { for } r_{\mathrm{c}}>0.1,(13)
\end{aligned}
$$

and $\chi^{2} /$ d.o.f. $=810.9 / 38$ and $199.4 / 37$, respectively. Thus the normalization factor for the small core clusters is larger than that for the large core clusters at the $10 \sigma$ significance level.

$-\beta-T$

There seems to be a trend of larger $\beta$ values for higher temperatures, as previously noted by Schindler (1999). However $R_{X Y}=0.27$ for 77 clusters, hence the correlation is not clear.

$-n_{\mathrm{e} 0}-T$

We see from Fig. 16d that there is a significant scatter of the central electron density, $n_{\mathrm{e} 0}$. We obtained $R_{X Y}=-0.28$ and thus the correlation is not clear from the data. On the other hand, we notice $n_{\mathrm{e} 0}$ is strongly correlated with the parameter $r_{\mathrm{c}}$, which will be discussed in the next subsection in more detail.

$-r_{500}-T$

A strong correlation between the overdensity radius and the temperature is found $\left(R_{X Y}=0.82\right)$, whose best-fit relation is:

$r_{500}\left[h_{70}^{-1} \mathrm{Mpc}\right]=0.38_{-0.02}^{+0.03}(k T)^{0.53_{-0.04}^{+0.04}}$,

and $\chi^{2} /$ d.o.f. is $151.2 / 75$. The power-law slope of $0.53 \pm 0.04$ is consistent with the value predicted from the self-similar model, 0.5 , within the error range.

- $M_{500}-T$

There is a tight correlation between the cluster mass and the temperature $\left(R_{X Y}=0.88\right)$. The best-fit relation is

$M_{500}\left[h_{70}^{-1} M_{\odot}\right]=1.64_{-0.26}^{+0.35} \times 10^{13}(k T)^{1.68_{-0.11}^{+0.10},}$

and $\chi^{2} /$ d.o.f. is $206.5 / 75$. The slope of $1.68_{-0.11}^{+0.10}$ is slightly steeper than that expected from the self-similar relation, i.e. $M_{500} \propto T^{1.5}$. However, from Fig. 16f, we consider that 
the degree of the departure from the self-similar relation is marginal if compared to the typical size of the statistical error bars of the data points.

We compare the $M_{500}-T$ relation with those derived for two samples of nearby clusters (Table 1 of Finoguenov et al. 2001). Because they calculated it under a different cosmology: $\Omega_{0}=1$ and $H_{0}=50 \mathrm{~km} \mathrm{~s}^{-1} \mathrm{Mpc}^{-1}$, we calculate the relation using the same set of cosmological parameters and obtain $M_{500}=2.04_{-0.38}^{+0.37} \times 10^{13}(k T)^{1.65_{-0.09}^{+0.11}}$ for the distant clusters. In comparison to their flux-limited sample (HIFLUGCS) and the sample with temperature profiles, the slope is in a good agreement within the errors but it should be noted that the best-fit normalization is about $30 \%$ smaller for the distant sample. This may be attributed to the significant redshift dependency of the critical density and will be worth further investigation in the light of the cluster formation redshift.

We also showed the $E_{z} M-T$ relation calculated for $\Delta_{\mathrm{c}}=$ $500 \Omega^{0.427}$ in Table 9. The result is within a range consistent with the relation for $z>0.4$ (Ettori et al. 2004).

- $M_{\text {gas }}-T$

The gas mass within $r_{500}, M_{\text {gas }}$, is strongly correlated to the temperature $\left(R_{X Y}=0.61\right)$. The fitting gives

$M_{\text {gas }}\left[h_{70}^{-5 / 2} M_{\odot}\right]=2.88_{-0.64}^{+0.93} \times 10^{12}(k T)^{1.85_{-0.15}^{+0.14}}$,

and $\chi^{2} /$ d.o.f. is $548.1 / 75$. Taking into account the current statistical errors, we found a marginal steepening of the relation in comparison to that predicted from the self-similar model, $M_{\text {gas }} \propto T^{1.5}$ (see Fig. $16 \mathrm{~g}$ ). The measured slope is also found to be consistent with that of the $M_{500}-T$ relation derived above within their errors.

For nearby clusters, Mohr et al. (1999) obtained the relation to be $M_{\text {gas }}=(1.49 \pm 0.09) \times 10^{14}(k T / 6 \mathrm{keV})^{1.98 \pm 0.18}$ under $\Omega_{0}=1$ and $H_{0}=50 \mathrm{~km} \mathrm{~s}^{-1} \mathrm{Mpc}^{-1}$, which is significantly steeper than the theoretically expected slope of 1.5 . On the other hand, Vikhlinin et al. (1999) reported based on the ROSAT PSPC data analysis of nearby regular clusters, a flatter relation in the form of $M_{\text {gas }} \propto T^{1.71 \pm 0.13}$, utilizing a different method in determining the limiting radius (they defined the baryon overdensity radius of $R_{1000}$, corresponding to the dark matter overdensity of $\sim 500$ ).

If we calculate the distant $M_{\text {gas }}-T$ relation within $r_{500}$ using the same set of cosmological parameters, $M_{\mathrm{gas}}=$ $4.57_{-1.17}^{+1.58} \times 10^{12}(k T)^{1.86_{-0.16}^{+0.16}}$. The relation is slightly flatter than that derived by Mohr et al. (1999) but within a range consistent with the result of either Mohr et al. (1999) or Vikhlinin et al. (1999) under the current measurement errors.

As shown above, although the relation within $r_{500}$ obtained for our distant sample is consistent with the local relations, it is found to be less steep than that found for $z>0.4$, $E_{z} M_{\text {gas }} \propto T^{2.37 \pm 0.17}$ (Ettori et al. 2004), which is not conflict with the view that the lower redshift clusters contain more gas for a fixed temperature, as pointed by Ettori et al. (2004).

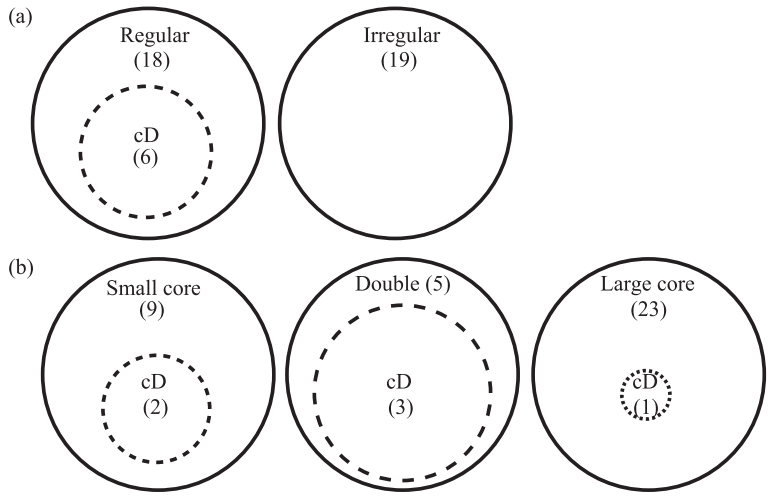

Fig. 17. Venn diagrams which illustrate the relation between the X-ray morphology and the optical morphology a) and the $\mathrm{X}$-ray core radius and the optical morphology b) (see Sect. 6.2 for definition of the $\mathrm{cD}$ cluster). In the panel $\mathbf{b}$ ), we divided the sample into three subgroups: small core $\left(r_{\mathrm{c}}<0.1 \mathrm{Mpc}\right)$ single- $\beta$, double- $\beta$, and large core $\left(r_{\mathrm{c}}>\right.$ $0.1 \mathrm{Mpc})$ single- $\beta$ clusters.

\section{$-f_{\text {gas }}-T$}

We obtained a small correlation coefficient, $R_{X Y}=-0.34$. The power-law fitting resulted in

$$
f_{\text {gas }}\left[h_{70}^{-3 / 2}\right]=0.28_{-0.06}^{+0.09}(k T)^{-0.08_{-0.14}^{+0.14}}
$$

and $\chi^{2} /$ d.o.f. $=349.8 / 75$. Because the resultant slope includes 0 within the error, we conclude that there is not a significant temperature dependence in the distant sample. Thus we show the mean gas-mass fraction of the sample, $\left\langle f_{\text {gas }}\right\rangle=0.20$, in Fig. $16 \mathrm{~h}$.

In the above analysis, we have not included the effect of the cluster redshift. However, because a weak redshift dependence is seen in the overdensity radius, which is $r_{500} \propto\left(1+z_{\text {obs }}\right)^{-0.6}$ (see Sect. 5.2.3), we checked how the parameter correlations in Eqs. (14)-(17) will be changed if we divide the sample into the low- $z(0.1<z \leq 0.3)$ and the high- $z(0.3<z<0.82)$ subsamples. As a result, there are no significant changes in the scaling relations compared to Eqs. (14)-(17) except that the $M_{\text {gas }}-T$ for the high- $z$ subsample resulted in a marginally steeper slope of $M_{\text {gas }}=5.4_{-4.0}^{+11.2} \times 10^{11}(k T)^{2.67_{-0.58}^{+0.70}}\left(\chi^{2} /\right.$ d.o.f. $\left.=170.2 / 30\right)$. In order to place firmer constraints on the scaling relations, we suggest that it is important to gather more observational data with higher sensitivities and also reexamine the assumption of the isothermal gas distribution and the formation redshift, $z_{\mathrm{col}}$ as already pointed out in Sect. 5.2.3.

\subsection{Correlations with the core radius}

- (Optical morphology) $-r_{\mathrm{c}}$

First we investigate the relation to the optical morphology of the clusters. Some clusters contain a central dominant elliptical galaxy, i.e. a cD galaxy. We refer to such clusters classified as Bautz-Morgan types I and I-II as "cD clusters". We looked up the BM types of our sample clusters in the NED database and showed the relation between X-ray morphology and the BM type, and the core radius and the BM type in Figs. 17a and 17b respectively. In Fig. 17b we 
(a)

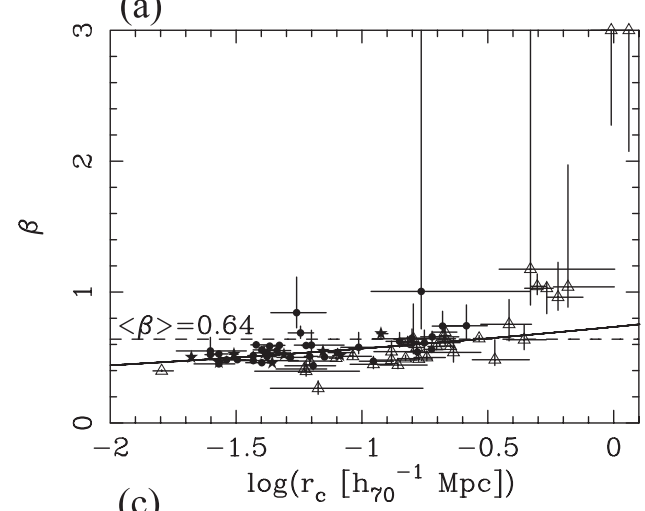

(c)

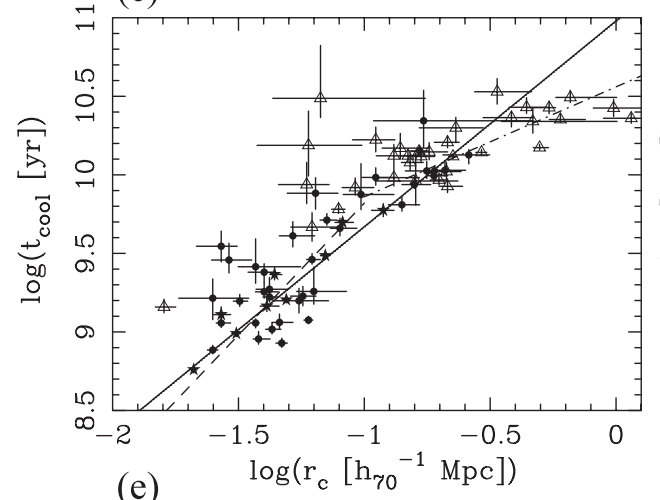

(e)

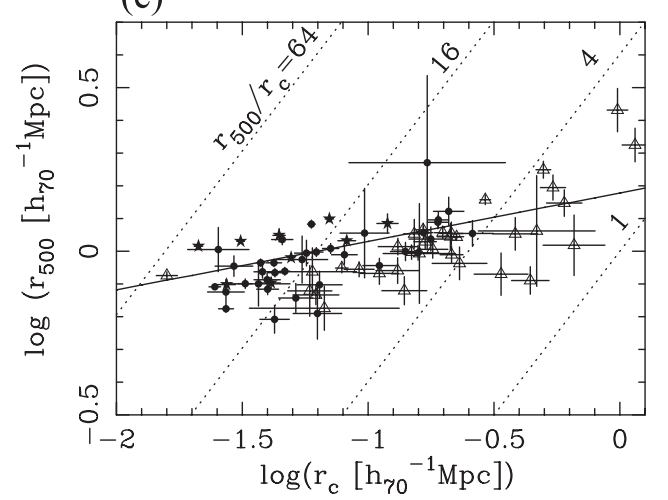

(b)

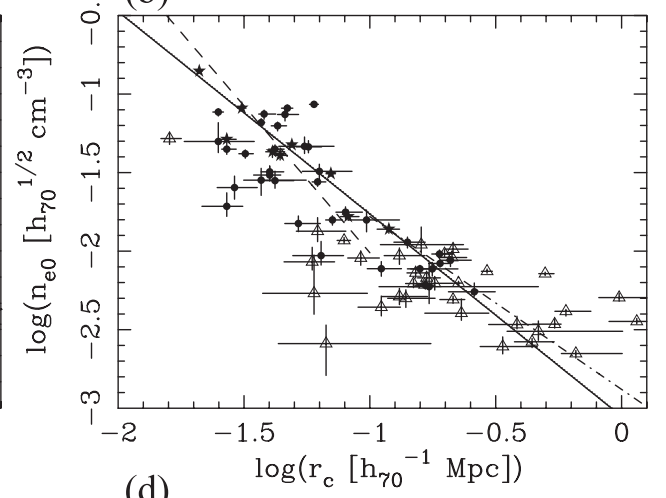

(d)

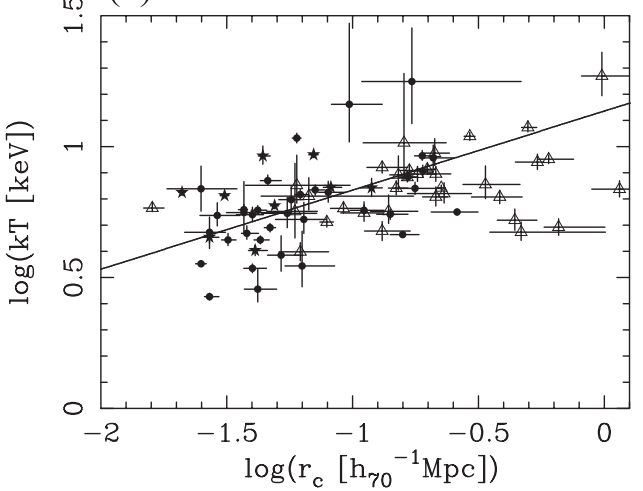

Fig. 18. Relations of $\beta$ a), the central electron density b), the cooling timescale c), the temperature d), and the overdensity radius e) with the core radius. The meanings of the symbols are the same as Fig. 16. The error bars are $1 \sigma$. In the panels a)-e) the best-fit power-laws obtained for 77 distant clusters are shown with the solid lines. In the panels b) and $\mathbf{c}$ ), the best-fit power-laws for log $r_{\mathrm{c}} \leq-1$ and $\log r_{\mathrm{c}}>-1$ are also shown with the dashed lines and the dot-dash lines, respectively. In the panel e) four dotted lines correspond to four different constant values of $r_{500} / r_{\mathrm{c}}$.

divided the clusters into three subgroups: the small core single- $\beta$, the large core single- $\beta$ and the double- $\beta$ clusters. We find that all the $\mathrm{cD}$ clusters are regular clusters and the clusters with a small core (i.e. small core single- $\beta+$ double- $\beta$ ) tend to contain a $\mathrm{cD}$ galaxy. However, not all the small core clusters have $\mathrm{cD}$ galaxies, and the fraction having a cD galaxy is $36 \%$ (5 of 14). Therefore it is not a simple one-to-one correspondence. In nearby clusters, the typical X-ray core radius of $\mathrm{cD}$ galaxy is measured to be $\sim 10 \mathrm{kpc}$ (Ikebe et al. 1999) and is significantly smaller than $50 \mathrm{kpc}$. Thus it is unlikely that the small core represents the potential distribution of the $\mathrm{cD}$ galaxy itself, though some connection may be possible. At present, however, the data of the optical morphology is available for only $47 \%$ of the sample. Thus in order to clarify the correlation between the central galaxy and the formation of the small core, we need to collect more optical data. Though the above discussion was based on the BM-type classification, we suggest that it is also meaningful to take into account the existence of giant ellipticals at the cluster center.

$-\beta-r_{\mathrm{c}}$

There is a weak trend of larger $\beta$ for larger $r_{\mathrm{c}}\left(R_{X Y}=0.62\right)$. The fit yields

$\beta=0.73_{-0.05}^{+0.07} r_{\mathrm{c}}^{0.11_{-0.02}^{+0.03}}$,

and $\chi^{2} /$ d.o.f. is $711.1 / 75$. However the fit is not statistically acceptable due to the huge $\chi^{2}$ value. Regarding this, we have to be careful about the parameter coupling in the $\beta$ model fitting because the correlation seems to follow the direction of the coupling (Fig. 5). This occurs noticeably at 
$r_{\mathrm{c}} \gtrsim 0.1 \mathrm{Mpc}$. The correlation is strong for the large core clusters with $r_{\mathrm{c}}>0.1 \mathrm{Mpc}\left(R_{X Y}=0.72\right)$ while it is weak $\left(R_{X Y}=0.09\right)$ for the small core clusters with $r_{\mathrm{c}}<0.1 \mathrm{Mpc}$. In addition, all the clusters that exhibit extremely large core radii $\left(r_{\mathrm{c}} \gtrsim 0.4 \mathrm{Mpc}\right)$ are irregular clusters and their surface brightness distributions are highly inhomogeneous or bimodal (Fig. C.1). Accordingly the current spherical $\beta$-model can cause the tight $\beta-r_{\mathrm{c}}$ coupling particularly for the irregular systems.

$-n_{\mathrm{e} 0}-r_{\mathrm{c}}$

As is clear from Fig. 14c, the central electron density, $n_{\mathrm{e} 0}$, has a double-peaked distribution, similar to $r_{\mathrm{c}}$. We see from Fig. 18b that there is a strong correlation between $n_{\mathrm{e} 0}$ and $r_{\mathrm{c}}$. The correlation coefficient is $R_{X Y}=-0.85$ for 77 clusters. From the $\chi^{2}$ fitting, we obtain

$n_{\mathrm{e} 0}\left[h_{70}^{1 / 2} \mathrm{~cm}^{-3}\right]=0.89_{-0.23}^{+0.30} \times 10^{-3} r_{\mathrm{c}}^{-1.29_{-0.11}^{+0.10}}$.

$\chi^{2} /$ d.o.f. is $1185 / 75$. We also find that the slope tends to be steeper for the small $r_{\mathrm{c}}$ clusters: if we fit the data points for two different $r_{\mathrm{c}}$ ranges separately, we obtain

$n_{\mathrm{e} 0}\left[h_{70}^{1 / 2} \mathrm{~cm}^{-3}\right]=0.13_{-0.09}^{+0.36} \times 10^{-3} r_{\mathrm{c}}^{-1.87_{-0.37}^{+0.41}}$ for $r_{\mathrm{c}} \leq 0.1$,

$n_{\mathrm{e} 0}\left[h_{70}^{1 / 2} \mathrm{~cm}^{-3}\right]=1.32_{-0.83}^{+1.30} \times 10^{-3} r_{\mathrm{c}}^{-1.10_{-0.66}^{+0.43}}$ for $r_{\mathrm{c}}>0.1$.

$\chi^{2} /$ d.o.f. are $650.3 / 39$ and $373.4 / 34$, respectively. Thus the gas distribution in clusters with $r_{\mathrm{c}} \leq 0.1 \mathrm{Mpc}$ is concentrated more than expected from the relation for clusters with $r_{\mathrm{c}}>0.1 \mathrm{Mpc}$. This may suggest that the small core and the large core components have different physical natures and/or they are at different stages of evolution.

$-t_{\text {cool }}-r_{\mathrm{c}}$

According to Figs. $12 \mathrm{e}$ and $18 \mathrm{c}, t_{\text {cool }}$ is significantly shorter than the age of the Universe for the small core clusters and then the radiative cooling is suggested to be important. We find a very tight correlation between $t_{\text {cool }}$ and $r_{\mathrm{c}}\left(R_{X Y}=\right.$ 0.87 for 77 clusters). The $t_{\text {cool }}-r_{\mathrm{c}}$ relations are derived to be

$$
\begin{aligned}
& t_{\text {cool }}[\mathrm{yr}]=9.55_{-2.07}^{+2.23} \times 10^{10} r_{\mathrm{c}}^{1.31_{-0.09}^{+0.08}} \text { for } 77 \text { clusters, } \\
& t_{\text {cool }}[\mathrm{yr}]=31.6_{-20.4}^{+51.1} \times 10^{10} r_{\mathrm{c}}^{1.68_{-0.32}^{+0.29}} \text { for } r_{\mathrm{c}} \leq 0.1, \\
& t_{\text {cool }}[\mathrm{yr}]=3.63_{-0.91}^{+1.21} \times 10^{10} r_{\mathrm{c}}^{0.70_{-0.18}^{+0.18}} \text { for } r_{\mathrm{c}}>0.1 .
\end{aligned}
$$

$\chi^{2} /$ d.o.f. are 690.0/75, 402.4/39 and 175.9/34, respectively. Thus one possible interpretation of the small core may be that the small core radius does not reflect the shape of the gravitational potential but that it reflects the cooling radius inside which the X-ray emission is enhanced. However we consider this is unlikely for the following reasons. If the small core radius reflects the cooling radius, it should evolve with time. However we do not find a strong redshift dependence in the core radius. Moreover we estimated the cooling radius at which the cooling time of the gas becomes equal to $t_{\text {age }}$ to find it is larger than $50 \mathrm{kpc}$ for most of the small core clusters.

$-T-r_{\mathrm{c}}$

Furthermore, as shown in Fig. 18d, we find that the temperature does not show a clear core radius dependence.
We obtained a moderate correlation coefficient, $R_{X Y}=$ 0.45 . The best-fit relation is derived as

$k T=13.7_{-2.5}^{+2.6} r_{\mathrm{c}}^{0.30_{-0.07}^{+0.05},}$

however, the power-law fit was quite poor $\left(\chi^{2} /\right.$ d.o.f. $=$ $2228 / 75)$. Since the emission-weighted temperature reflects the temperature of the cluster core region, the apparent lack of any strong $T-r_{\mathrm{c}}$ correlation suggests that the temperature gradient in the cooling region is not very large. This is consistent with the results from the XMM-Newton observations, which revealed that the temperature gradient of nearby cooling-flow clusters is smaller than that expected from the standard cooling flow model (e.g. Tamura et al. 2001). Peterson et al. (2003) noted that there is a significant deficit of emission with temperature lower than $T_{0} / 3$ ( $T_{0}$ is the ambient temperature) in the RGS spectra. Given that the temperature profile obeys $T(r) \propto r^{0.2}$, the second term in Eq. (4) is negligible compared to the first term at $r \gtrsim 0.3 r_{\mathrm{c}}$ for $\beta=2 / 3$. Thus except for the central $r \lesssim 0.3 r_{\mathrm{c}}$ region, the cluster mass profile can be approximated with the assumption of a constant temperature, suggesting the gas density profile obtained from the current isothermal $\beta$-model analysis reflects the underlying cluster potential distribution. We further investigate the relation between $r_{500}$ and the core radius below.

- $r_{500}-r_{\mathrm{c}}$

Since the overdensity radius is determined almost independently from $r_{\mathrm{c}}$ and approximately $r_{500} \propto T^{1 / 2}$, the above results indicate that correlation between $r_{500}$ and $r_{\mathrm{c}}$ is much weaker than the expectations of the self-similar model. It is clear from Fig. 18e that the distribution of the data points are inconsistent with the curves of $r_{500} / r_{\mathrm{c}}=$ constant. The observed range of $r_{500} / r_{\mathrm{c}}$ is about $2-40$. The correlation coefficient is $R_{X Y}=0.58$ for 77 clusters. Then the $\chi^{2}$ fitting gives

$r_{500}\left[h_{70}^{-1} \mathrm{Mpc}\right]=1.51_{-0.17}^{+0.16} r_{\mathrm{c}}^{0.15_{-0.04}^{+0.03}}$ for 77 clusters,

and $\chi^{2} /$ d.o.f. $=954.5 / 75$. We notice that the observed distribution on the $r_{500}-r_{\mathrm{c}}$ plane is much flatter than the curves for constant $r_{500} / r_{\mathrm{c}}$ values or it is rather concentrated around the two peak values of $r_{\mathrm{c}}$. The departure from the self-similar relation is more prominent for small core clusters with $r_{\mathrm{c}}<0.1 \mathrm{Mpc}$. The correlation coefficients are $R_{X Y}=0.21$ and 0.36 for $r_{\mathrm{c}} \leq 0.1$ and $r_{\mathrm{c}}>0.1$, respectively. Thus for the large core clusters, the best-fit power-law relation is

$r_{500}\left[h_{70}^{-1} \mathrm{Mpc}\right]=2.06_{-0.33}^{+0.42} r_{\mathrm{c}}^{0.37_{-0.11}^{+0.12}}$ for $r_{\mathrm{c}}>0.1$,

and $\chi^{2} /$ d.o.f. is $108.0 / 34$. If we further restrict $r_{\mathrm{c}}$ to a very narrow range of $0.1-0.2 \mathrm{Mpc}$, we find a steeper slope of $r_{500}=4.03_{-1.52}^{+3.22} r_{\mathrm{c}}^{0.71_{-0.26}^{+0.30}}\left(\chi^{2} /\right.$ d.o.f. $\left.=21.2 / 18\right)$. Thus those twenty clusters may satisfy the self-similar condition, $r_{500} \propto r_{\mathrm{c}}$. However, we suggest from Eqs. (26) and (27) that it is difficult to explain the formation of the cores, particularly for the small core clusters, by the standard picture of the self-similar model. 


\subsection{Implications on the origin of two core scales}

From the above discussion, it seems difficult to explain the small core size that we discovered in the histogram either by the potential structure of the $\mathrm{cD}$ galaxy or the cooling radius. As long as we rely on the hydrostatic assumption and the $\beta$ model, the X-ray surface brightness distributions are likely to represent the gravitational potential structures of the clusters. If this is the case, the double- $\beta$ nature of the X-ray emission profile reflects the shape of the gravitational potential of the dark matter, which is likely to have two preferable scales of $\sim 50 \mathrm{kpc}$ and $\sim 200 \mathrm{kpc}$.

In an effort to constrain the physical status of ICM in the dark matter potential, comparing the high-resolution X-ray observations to gravitational lensing observations will provide another powerful test (e.g. Hattori et al. 1997). Thanks to the improvement of spatial resolution achieved by Chandra, now measurements on the cluster mass profile down to $\lesssim 5 \mathrm{kpc}$ scale are possible at such high redshifts $(z \sim 0.3)$ and several authors have measured the dark matter distribution in the lensing clusters under the hydrostatic hypothesis (e.g. Arabadjis et al. 2002; Xue \& Wu 2002; Ota et al. 2004). For example, Ota et al. (2004) showed from the high-resolution Chandra data of CL0024+17 $(z=0.395)$ and the comparison with the detailed lens modeling by Tyson et al. (1998) that the cluster density profile is well reproduced by the double- $\beta$ model and the inner core also reflects the underlying dark matter potential. They also noted that the core structure may be related to the past merging event as inferred from the optical observations (Czoske et al. 2001, 2002).

Recently Hayakawa et al. (2004) estimated the dark matter distribution in a nearby non-cD, regular cluster, Abell 1060, from the Chandra data analysis, without explicitly using the double $\beta$-model, and found a central mass concentration at $r<50 \mathrm{kpc}$. Their result also supports the idea that dark matter may preferentially be accumulated within a radius of $\sim 50 \mathrm{kpc}$. On the other hand, Ettori et al. (2004) suggested that no significant double structure is seen in the high redshift sample. Thus considering from the above, the double- $\beta$ nature of the ICM discovered in the present sample may be much related to the history of the past merging and the relaxation process. The small core component might be attributed to the presence of dark matter subhalos due to the cluster mergers or the internal structures in clusters (e.g. Fujita et al. 2002).

Furthermore, since there is clearly a tight coupling between the core radius and the radiative cooling time as shown in Sect. 6.2, the detailed treatment of the thermal evolution of the ICM will also be important. A number of numerical simulations including non-gravitational effects such as radiative cooling and galaxy feedback have been carried out and thus provide a clue to the underlying physics in the cluster core regions. However, the authors pointed out difficulties in regulating the central over-cooling and produce a constant-density core (e.g. Pearce et al. 2000). Masai \& Kitayama (2004) recently proposed a quasi-hydrostatic model, which predicts a characteristic temperature profile with an asymptotic temperature for the central region being $\sim 1 / 3$ of the non-cooling outer region, as observed in nearby "cooling flow" clusters. Thus detailed comparison of the X-ray data with their model regarding the temperature and density profiles will be important to understand the evolution of the ICM structure. We need further investigations to put a stronger constraint on the origin of the double nature of the cluster structures, which is however beyond the scope of the present paper and will be discussed in a separate paper.

\section{Summary}

We have analyzed the ROSAT HRI and the ASCA GIS/SIS data of 79 clusters of galaxies at redshifts of 0.1-0.82 in a uniform manner. We determined the X-ray surface brightness profile from the ROSATHRI data utilizing the $\beta$-model and the average temperature and the luminosity from the ASCA data. We found that the clusters can be divided into two subgroups, regular and irregular clusters, from analysis to determine the centroid position of the X-ray image. We then performed a statistical study of the X-ray parameters and investigated the trends for redshift evolution and the scaling relations against temperature and core radius. The major results are summarized as follows.

1. We did not find significant redshift evolution in the X-ray parameters of clusters compared to the nearby clusters: the temperature $k T$, the core radius $r_{\mathrm{c}}, \beta$, and the central electron density $n_{\mathrm{e} 0}$ at $z \lesssim 0.5$.

2. Among the $X$-ray parameters, the core radius shows the largest cluster-to-cluster variation. The core-radius distribution shows two distinct peaks at $50 \mathrm{kpc}$ and $200 \mathrm{kpc}$. For $20 \%$ of the regular clusters, inclusion of a second $\beta$-model component significantly improved the $\chi^{2}$ values of the surface-brightness fitting. We find that the two core radii of the double $\beta$-model are distributed in relatively narrow ranges consistent with the two peaks of the single $-\beta$ clusters. There is no significant evolution in the fraction of double- $\beta$ clusters within the observed redshift range.

3. We investigated the correlations between the temperature and the cluster parameters including the spectral and the $\beta$-model parameters, the cluster mass, the gas mass and the gas-mass fraction etc. For the $M_{500}-T$ relation, we found that the power-law slope of $1.68_{-0.11}^{+0.10}$ is marginally steeper than that expected from the self-similar model but in a good agreement with the results for the nearby clusters, while the normalization factor is about $30 \%$ smaller for the current distant sample compared to the nearby sample. We obtained the $M_{\text {gas }}-T$ relation to be $M_{\text {gas }} \propto(k T)^{1.86_{-0.16}^{+0.16}}$ for the overdensity of $\Delta_{\mathrm{c}}=500$ and found a marginal steepening of the relation in comparison to the self-similar model under the current statistics. The $f_{\text {gas }}-T$ relation is found to be consistent with having no correlation with the temperature.

4. We studied the parameter correlations against the core radius. We found that only $36 \%$ of the small core single $-\beta$ and the double- $\beta$ clusters are $\mathrm{cD}$ clusters and thus it seems difficult to explain the presence of the small core by the $\mathrm{cD}$ potential itself although there may be some causal link. There are strong $n_{\mathrm{e} 0}-r_{\mathrm{c}}$ and $t_{\mathrm{cool}}-r_{\mathrm{c}}$ correlations and the slopes tend to become steeper for $r_{\mathrm{c}} \lesssim 0.1 \mathrm{Mpc}$. On the other hand the fact that there is not a clear $T-r_{\mathrm{c}}$ 
correlation suggests that the temperature gradient is not large even in clusters with short cooling timescales, which is consistent with the XMM-Newton and Chandra observations of the nearby clusters. Thus as long as we rely on the hydrostatic condition and the $\beta$-model, our result indicates that the dark matter distribution is likely to show two preferable scales of $50 \mathrm{kpc}$ and $200 \mathrm{kpc}$.

5. We showed that the $r_{500}-r_{\mathrm{c}}$ relation derived from the $\mathrm{X}$-ray analysis does not agree with the expectations of the self-similar model, suggesting that the assumption of selfsimilarity is not valid in describing the density profile of the ICM, particularly for clusters with small core radius.

6. We obtained the average gas-mass fraction within $r_{500}$ to be $\left\langle f_{\text {gas }}\right\rangle=(0.20 \pm 0.07) h_{70}^{-3 / 2}$ for the distant sample. The calibrational error is estimated to be about $25 \%$. The current estimation is based on some simplified assumptions (for example, the isothermal gas distribution), which will be refined in future studies.

Acknowledgements. We are grateful to S. Sasaki, T. Kitayama and K. Masai for their helpful comments and discussions. N.O. is supported by a Research Fellowship for Young Scientists from the JSPS. This research has made use of the ROSAT HRI data obtained through the ROSAT Data Archive of MPE at Garching, Germany and the ASCA data through the DARTS Online Service provided by ISAS (ISAS/JAXA) and the HEASARC Online Service provided by NASA/Goddard Space Flight Center. We also thank the anonymous referee for helpful comments.

\section{References}

Allen, S. W. 1998, MNRAS, 296, 392

Arabadjis, J. S., Bautz, M. W., \& Garmire, G. P. 2002, ApJ, 572, 66

Arnaud, K. A. 1996, ASP Conf. Ser., 101, Astronomical Data Analysis Software and Systems V, 5, 17

Arnaud, M., \& Evrard, A. E. 1999, MNRAS, 305, 631

The ASCA Data Reduction Guide 2002, ASCA Guest Observer Facility (http://heasarc.gsfc.nasa.gov/docs/asca/abc/ abc.html)

Briel, U. G., et al. 1997, The ROSAT User's Handbook, (http://wave.xray.mpe.mpg.de/rosat/doc/ruh)

Cavaliere, A., \& Fusco-Femiano, R. 1976, A\&A, 49, 137

Cavaliere, A., Menci, N., \& Tozzi, P. 1997, ApJ, 484, L21

Chartas, G., Bautz, M., Garmire, G., Jones, C., \& Schneider, D. P. 2001, ApJ, 550, L163

Czoske, O., Kneib, J.-P., Soucail, G., et al. 2001, A\&A, 372, 391

Czoske, O., Moore, B., Kneib, J.-P., \& Soucail, G. 2002, A\&A, 386, 31

David, L. P., Slyz, A., Jones, C., et al. 1993, ApJ, 412, 479

Edge, A. C., Stewart, G. C., Fabian, A. C., \& Arnaud, K. A. 1990, MNRAS, 245, 559

Ettori, S., \& Fabian, A. C. 1999, MNRAS, 305, 834

Ettori, S., Tozzi, P., Borgani, S., \& Rosati, P. 2004, A\&A, 417, 13

Evrard, A. E., \& Henry, J. P. 1991, ApJ, 383, 95

Evrard, A. E., Metzler, C. A., \& Navarro, J. F. 1996, ApJ, 469, 494

Finoguenov, A., Reiprich, T. H., \& Böhringer, H. 2001, A\&A, 368, 749
Fujita, Y., Sarazin, C. L., Nagashima, M., \& Yano, Y. 2002, ApJ, 577, 11

Hashimotodani, K. 1999, Ph.D. Dissertation, Osaka University Hattori, M., Ikebe, Y., Asaoka, I., et al. 1997, Nature, 388, 146

Hattori, M., Kneib, J.-P., \& Makino, N. 1999, Prog. Theor. Phys. Suppl., 133, 1

Hayakawa, A., Furusho, T., Yamasaki, N.Y., Ishida, M., \& Ohashi, T., PASJ, submitted

Ikebe, Y., Makishima, K., Fukazawa, Y., et al. 1999, ApJ, 525, 58

Jones, C., \& Forman, W. 1984, ApJ, 276, 38

Kaiser, N. 1986, MNRAS, 222, 323

Lewis, A. D., Ellingson, E., Morris, S. L., \& Carlberg, R. G. 1999, ApJ, 517, 587

Masai, K., \& Kitayama, T. 2004, A\&A, 421, 815

Markevitch, M. 1998, ApJ, 504, 27

Mohr, J. J., Fabricant, D. G., \& Geller, M. J. 1993, ApJ, 413, 492

Mohr, J. J., Mathiesen, B., \& Evrard, A. E. 1999, ApJ, 517, 627

Mushotzky, R. F., \& Loewenstein, M. 1997, ApJ, 481, L63

Mushotzky, R. F., \& Scharf, C. A. 1997, ApJ, 482, L13

Nakamura, T. T., \& Suto, Y. 1997, Prog. Theor. Phys., 97, 49

Neumann, D. M., \& Arnaud, M. 2001, A\&A, 373, L33

Novicki, M. C., Sornig, M., \& Henry, J. P. 2002, AJ, 124, 2413

Ota, N. 2001, Ph.D. Thesis, University of Tokyo (ISAS Research Note No. 727)

Ota, N., \& Mitsuda, K. 2002, ApJ, 567, L23

Ota, N., Mitsuda, K., \& Fukazawa, Y. 1998, ApJ, 495, 170

Ota, N., Mitsuda, K., Hattori, M., \& Mihara, T. 2000, ApJ, 530, 172

Ota, N., Pointecouteau, E., Hattori, M., \& Mitsuda, K. 2004 ApJ, 601, 120

Pearce, F. R., Thomas, P. A., Couchman, H. M. P., \& Edge, A. C. 2000, MNRAS, 317, 1029

Peterson, J. R., et al. 2003, ApJ, 590, 207

Prestwitch, A. H., Silverman, J., McDowell, J., Callanan, P., \& Snowden, S. 1998, Spectral Calibration of the ROSAT HRI (http://hea-www . harvard.edu/rosat/rsdc_www/HRISPEC/ spec_calib.html)

Raymond, J. C., \& Smith, B. W. 1977, ApJS, 35, 419

Sanderson, A. J. R., Ponman, T. J., Finoguenov, A., Lloyd-Davies, E. J., \& Markevitch, M. 2003, MNRAS, 340, 989

Schindler, S. 1999, A\&A, 349, 435

Schmidt, R. W., Allen, S. W., \& Fabian, A. C. 2001, MNRAS, 327, 1057

Snowden, S. L. 1998, ApJS, 117, 233

Soucail, G., Ota, N., Bohringer, H., Czoske, O., Hattori, M., \& Mellier, Y. 2000, A\&A, 355, 433

Spergel, D. N., Verde, L., Peiris, H. V., et al. 2003, ApJS, 148, 175

Tanaka, Y., Inoue, H., \& Holt, S. S. 1994, PASJ, 46, L37

Tamura, T., Kaastra, J. S., Peterson, J. R., et al. 2001, A\&A, 365, L87

Trümper, J. 1993, Science, 260, 1769

Tyson, J. A., Kochanski, G. P., \& Dell'Antonio, I. P. 1998, ApJ, 498, L107

Vikhlinin, A., Forman, W., \& Jones, C. 1999, ApJ, 525, 47

Vikhlinin, A., VanSpeybroeck, L., Markevitch, M., Forman, W. R., \& Grego, L. 2002, ApJ, 578, L107

Xue, S. J., \& Wu, X. P. 2002, ApJ, 572, 152

White, D. A. 2000, MNRAS, 312, 663

Yaqoob, T. 1999, ApJ, 511, L75

Zimmermann, H.-U., et al. 1992, in Data Analysis in Astronomy IV, ed. V. Di. Gesu, L. Scarsi, R. Buccheri, P. Crane, M. Maccarone, \& H.-U. Zimmermann (New York, London: Plenum Press), 141 